\title{
COSTRUIRE INGEGNOSAMENTE RIUTILIZZANDO MATERIALI POVERI. L'IMPIEGO DI CONCHIGLIE A FINI EDILIZI AD AOUILEIA TRA ETÀ REPUBBLICANA E TARDA ANTICHITÀ
}

\author{
Simone Dilaria \\ s.dilaria88@gmail.com \\ Dipartimento di Beni Culturali - Università degl Studi di Padova
}

\begin{abstract}
In this paper, we take into exam six different contexts in Roman Aquileia in which considerable amounts of shells, originally collected for different purposes (dietary consumption or purple dye production), were re-used as raw material in building activities, either inserted in mortars or used as floor foundation. Shells as building material have useful properties, which are here analyzed. Themes such as technological transmission among cultures are discussed through the examination of other attestations of these practices in the ancient Mediterranean.
\end{abstract}

\section{INTRODUZIONE}

Il rinvenimento di malacofauna negli scavi archeologici non rappresenta, di per sé, un fenomeno raro. Conchiglie sono di frequente ritrovate in concentrazioni di pochi esemplari sporadici o, più raramente, possono presentarsi in accumuli considerevoli, il cui processo formativo può essere naturale o antropico ${ }^{1}$.

Diversi sono i motivi per cui l'uomo si procacciava e impiegava le conchiglie in antico: primo tra tutti è sicuramente l'alimentazione ${ }^{2}$. Secondariamente, conchiglie potevano essere impiegate per scopi rituali, decorativi (sotto forma di monili e pendenti in collane e ceramiche), o per altri fini che spaziano dall'impiego come pesi in reti da pesca, all'uso come lucerne ${ }^{3}$. Infine, il rinvenimento di conchiglie di alcune specie di murice (Hexaplex Trunculus, Bolinus Brandaris o Thais Haemastoma), in stato frammentario o con incisioni nella parte centrale del guscio, rivela una particolare lavorazione, ricordata da Plinio il Vecchio (Nat. Hist. IX, 124-137), atta al prelievo di una ghiandola dall'animale dalla quale si ricava, fin dall'antichità, la porpora ${ }^{4}$.

Si possono quindi distinguere due forme di uso di malacofaune: primario nel caso in cui vengono raccolte intenzionalmente le conchi-

1 Reitz - Shackley, 2012, p. 360; Claassen, 1998, pp. 10-12; Girod, 2015, pp. 64-66.

2 Girod, 2015, pp. 43-45, 79-80, 96-100.

3 In generale si veda GIROD, 2015, pp. 45-61, 82-88, 100-102; per l'età del Bronzo in era egea v. KARALI, 1999, p. 43; per l'età ellenistica e romana v. REESE, 1979-1980, p. 81.

4 Sulle fasi di produzione della porpora v. Alberti, 2008, pp. 74-76; Karali, 1990, pp. 413-414; KARALI, 1999, pp. 43-44; ReESE, 1979-80, pp. 83; ReESE, 1987, p. 203; Ruscillo, 2005; Ruscillo, 2006, pp. 807-815; PedrazZi, 2011. 
glie mentre l'animale all'interno viene scartato; secondario quando, una volta raccolto l'animale a scopo edule o per attività artigianali, la conchiglia che lo conteneva viene reimpiegata per scopi diversi ${ }^{5}$. Tra le pratiche di reimpiego ("utilizzo secondario") vi è l'uso di conchiglie, una volta frantumate, come sgrassante/temprante negli impasti di ceramiche al fine di ottenere recipienti più solidi ${ }^{6}$. Più spesso potevano essere impiegate come materiale da costruzione. Gli impieghi di conchiglie in ambito edilizio sono prevalentemente quattro:

- in stesure di fondazione di strutture o preparazione di pavimenti, dove spesso si rinvengono intere;

- come aggregato nelle malte;

- come legante una volta calcinate, in virtù della loro natura carbonatica ${ }^{7}$;

- come incluso negli impasti a matrice terrosa dei rivestimenti pavimentali per migliorare la resistenza meccanica e la coesione dei piani di calpestio ${ }^{8}$.

Tuttavia, ricerche mirate all'uso a fine costruttivo di conchiglie sono state condotte, finora, solo di rado' ${ }^{9}$. Riferimenti alle forme di abbandono o di riuso di grosse quantità di gusci conchigliferi sono invece frequenti in letteratura in quanto forniscono indiretta testimonianza dei motivi che hanno spinto al loro originario approvvigionamento (consumo alimentare, produzione della porpora ${ }^{10}$ ). Cionondimeno una ricerca sulle modalità di uso secondario di conchiglie in ambito costruttivo può offrire anchessa testimonianza di tradizioni costruttive e trasmissioni di pratiche sociali tra diverse culture del Mediterraneo antico.

Dopo aver chiarito funzione, proprietà e modalità di impiego di conchiglie in ambito edilizio, verranno in primo luogo richiamati, da dati disponibili in letteratura, i contesti in cui tali pratiche sono state documentate nel bacino Mediterraneo. Verranno quindi presi in esame alcuni casi riscontrati ad Aquileia riferibili all'età romana, in un arco cronologico compreso tra età tardo Repubblicana e tarda antichità, in cui conchiglie sono state impiegate a fine costruttivo come preparazio-

5 Girod, 2015, p. 42.

6 Pratica attestata soprattutto in età protostorica. v. KARALI, 1990, p. 414; KARALI, 1999, p. 43; CARANnATE, 2014, pp. 275-277 e bibliografia citata. Non è altresì possibile dimostrare con certezza che non vi fosse in antico un approvvigionamento intenzionale (uso primario) di conchiglie da impiegare come sgrassante di ceramiche o per attività costruttive.

7 Su questi tre primi punti si tornerà più oltre. cfr. infra.

8 Cfr. nota 57.

9 Per alcuni studi incentrati sulle applicazioni di tali materiali al costruito cfr. Brysbaert, 2007; LAW, 2014; Ronen, 1980.

10 Una casistica degli indicatori sulla produzione della porpora in base alle forme di uso e riuso di malacofauna è in Alberti, 2008. 
ne per piani pavimentali, fondazione di strutture o come aggregato in malte.

\section{PROPRIETÀ ED IMPIEGO DI CONCHIGLIE NELLE MALTE}

Le malte e i calcestruzzi, da alcuni definiti come "miscele leganti" o, semplicemente, "leganti", sono dei composti artificiali realizzati dall'uomo e prodotti mescolando in acqua, per l'appunto, un legante, che è ciò che permette alla malta di far presa. Spesso composto da un materiale carbonatico (calce) o gesso, il legante è in genere miscelato con gli "aggregati", materiali, quali sabbia o frammenti fittili, che contrastano il ritiro del composto man mano che questo si solidifica, evitando così la formazione di fratture ${ }^{11}$.

Le conchiglie, in virtù della loro composizione carbonatica, possono svolgere la funzione di ottimi aggregati migliorando la coesione della malta ${ }^{12}$, soprattutto se fratturate ${ }^{13}$. Alcuni ritengono che venissero aggiunte volontariamente negli impasti per risparmiare sulla materia prima, pur avendo a disposizione un materiale robusto ma al contempo leggero e quindi facile da trasportare ${ }^{14}$. Ciò rendeva il tritume conchigliare particolarmente adatto per essere adoperato nei masselli preparatori di emblemata pavimentali che, come noto, essendo spesso prodotti per l'esportazione e trasportati per lunghe distanze entro cassette, necessitavano di supporti durevoli ma preferibilmente anche non troppo pesanti ${ }^{15}$.

La natura carbonatica delle conchiglie ne permette la calcinazione e quindi l'utilizzo non solo come aggregato ma anche come legante di malte $^{16}$. Tuttavia dimostrarne l'impiego attraverso sole analisi macroscopiche non è semplice ${ }^{17}$, a meno che non rimangano residui di fram-

11 Ginouvès - Martin, 1985, p. 48; Pecchioni et al., 2008.

12 Brysbaert, 2003, p. 175; Brysbaert, 2007, p. 43; Reese, 1987, p. 205.

13 Аїт KaCi et al., 2009, p. 234; Stefanidou et al., 2012, p. 749; Wilson - TÉBAR Megías, 2008, p. 233; Wootton, 2012, p. 221.

14 KaWERAU - Wiegand, 1930, pp. 53-54.

15 Westgate, 2000, p. 272, nota 66. Sulle tecniche produttive di emblemata pavimentali cfr. Dunbabin, 1999, pp. 288-290. Per F. Trapani la funzione di alleggerimento conferita dalle conchiglie nella malta è prioritaria rispetto al loro valore "cementante" (Trapani, 2012, p. 226).

16 Brysbaert, 2007; ReESe, 1979-80, p. 89-92; ReESe, 1987, p. 205.

17 Ciononostante diversi indicatori archeologici permettono di supporre l'impiego di conchiglie calcinate in funzione di legante: a Kommos in età medio minoica viene rilevata la presenza di accumuli di gusci di murex calcinati e pronti per essere impiegati nelle malte (Ruscillo, 2006, pp. 789, 802, 807-808, 816), inoltre calce prodotta calcinando murici sembra essere impiegata nei rivestimenti di Santorini e di Cipro dell'età del Bronzo (Ruscillo, 2006, p. 803); in età ellenistica a Kerkouane depositi di murici fracassati rinvenuti presso una fornace per la calce erano, con ogni probabilità, destinati alla calcinazione (FANTAR, 1984, p. 296; FAnTAR, 1986, p. 511); a Pergamo, in alcuni pavimenti del Palazzo V, le stesse conchiglie usate come aggregato nelle malte di supporto 
menti di gusci testimoni di una calcinazione non correttamente completata $^{18}$; arduo è pure dimostrare la calcinazione di conchiglie attraverso analisi archeometrica, in quanto la struttura mineralogica aragonitica dei gusci, una volta calcinati, si modifica in normale calcite, il cui abito cristallino non è dissimile da quello dei normali calcari carbonatici ${ }^{19}$. Comunque, qualora si rinvengano residui incotti, l'esito della cottura di sole conchiglie si può distinguere dalla calcinazione di calcari contenenti gusci fossili in quanto questi ultimi si dovrebbero presentare ancora ben cementati nella matrice litoide ${ }^{20}$.

Certo è che le qualità fisico-meccaniche offerte dall'impiego di conchiglie negli impasti di malte e intonaci dovevano essere ben note ai costruttori antichi. Il primo riferimento a tale la pratica è tuttavia attestato nelle fonti letterarie solo dalletà romana tardo antica: autore vissuto probabilmente nel IV d.C., Rutilio Tauro Palladio (Op. agr., I, 40, 3) suggerisce, in occasione di restauri dei rivestimenti di ambienti termali e bagni tiepidamente riscaldati, di aggiungere gusci opportunamente tritati (ostrei testae) negli impasti delle malte al fine di migliorare la presa del composto ${ }^{21}$.

Va comunque distinto l'impiego intenzionale in malte di conchiglie intere $\mathrm{o}$ in frammenti dalle occorrenze involontarie dovute, ad esempio, all'impiego, in funzione di aggregato, di sabbie prelevate da litorali fluviali o costieri e non opportunamente setacciate. Ciò fornisce comunque informazioni importanti sul luogo di approvvigionamento della materia prima ${ }^{22}$. Lanalisi della specie, del tasso di frattura e soprattutto della frequenza in presenza di conchiglie in una malta o calcestruzzo si

dovevano costituire, secondo coloro che le hanno studiate, anche il legante, in quanto, in seguito ad analisi chimica di quest'ultimo, sono state trovate tracce di acido fosforico di origine animale (KAWERAU - Wiegand, 1930, p. 54). Per l'età romana imperiale indizi su tale pratica provengono da Berenice (ReEse, 1979-80, pp. 87-92).

18 Brysbaert, 2007, p. 42. Anche in questo caso non è comunque possibile affermare che questi frammenti siano residui di attività di calcinazione e non siano invece solo aggregati, inseriti intenzionalmente o involontariamente nelle malte con funzione sgrassante.

19 BRysbaert, 2007, in part. pp. 42-43. La presenza di tracce aragonitiche in seguito ad analisi in diffrazione può essere dovuta esclusivamente alla non completa calcinazione di frammenti di guscio, che dovrebbero infatti convertirsi in calcite qualora cotti a temperature superiori ai $400-520^{\circ} \mathrm{C}$. Questi dovrebbero quindi essere ancora identificabili attraverso analisi in microscopia ottica o SEM.

20 Stefanidou et al., 2012, p. 749.

21 Greco, 2011, pp. 133, 135. L'utilizzo del termine testae, in genere utilizzato per i laterizi in frammenti, fa ritenere ad A.V. Greco che la preparazione cui dovevano essere oggetto le conchiglie da impiegare nelle malte dovesse seguire la stessa procedura adottata per frammentare materiali fittili.

22 LAW, 2014, pp. 245, 247. 
rivela quindi operazione necessaria per interpretarne l'occorrenza come intenzionale o meno, aspetto però solo di rado discusso in letteratura ${ }^{23}$.

Con queste premesse, verranno di seguito riportati e analizzati altri casi in cui sono stati documentati inclusi conchigliferi nelle malte, pur sussistendo la problematica legata alla difficoltà di dimostrarne, dai dati riportati in letteratura, l'intenzionalità di impiego (Tab. 1).

\section{Attestazioni nel Mediterraneo}

L'uso di conchiglie, intere o in frammenti, come aggregato in rivestimenti parietali e pavimentali è attestato nel Mediterraneo antico già dall'età del Bronzo in diversi siti di area Egea. Le prime attestazioni (MM - TM) provengono dai centri cretesi di Palaikastro, Kato Zakros, Chania, Knossos, Pseira, Vassiliki ${ }^{24}$ e dalle isole dell'Egeo tra cui Ayia Irini (Keos), Phylakopi (Milos), e Akrotiri (Santorini) ${ }^{25}$. Per quanto riguarda la Grecia continentale i contesti più antichi sono documentati a Tebe e Tirinto a cui si aggiunge Mileto in Asia Minore ${ }^{26}$. Leggermente più tardi, riusi di gusci di mollusco nelle malte sono attestati in contesti del tardo Bronzo in Levante ad Hala Sultan Tekke (Cipro), Sarepta (Libano), Lachish, Ascalona e, in Egitto, a Tell el-Dab'a, nelle malte preparatorie di affreschi di un edificio di un periodo forse contestuale alla diciottesima dinastia ${ }^{27}$. Nell'ultimo caso, iconografia, materiali (malta di calce al posto di malta di gesso) e messa in opera permettono di inserire appieno gli affreschi del sito egiziano nella koinè tecnico-produttivo di stampo egeo-orientale ${ }^{28}$. In tutti questi casi, le conchiglie, rinvenute in abbondanti quantità, sono sempre relative alle diverse specie di murice. Il dato è stato sempre sottolineato dagli autori in quanto offre diretta testimonianza della produzione della porpora.

23 Solo in alcuni casi viene discusso se l'impiego delle conchiglie nelle malte sia volontario o meno. Si veda ad esempio Wootton, 2012, p. 221; Coutelas, 2003, pp. 28-29, 149-150; STEFANIDOU et al., 2012, p. 749.

24 Alberti, 2008, in particolare tavv. 3-5, pp. 80 e 82-84; Brysbaert, 2002, pp. 103-104; Brysbaert, 2003 in particolare tav. XLIIb; BRysBaert, 2007, p. 32, tav. 1 con riferimenti bibliografici ivi riportati; REESE, 2016, pp. 409-412; in particolare su Palaikastro: Mac Gillivray et al., 1992, p. 126 e fig. a, plate 5; Knappett - Cunningham, 2012 , p. 85. Secondo D. Reese (2016, p. 412) a Knossos non sono stati individuati contesti in cui siano presenti frammenti di murex nelle malte, contrariamente a quanto riportato in BRYSBAERT, 2007, p. 32, tav. 1.

25 Per Ayia Irini v. Alberti, 2008, p. 80 e nota 21; per Phylakopi e Akrotiri v. Alberti 2008, p. 80; Brysbaert, 2003, in particolare tav. XLIIb; Brysbaert, 2007, p. 32 , tav. 1. Per Akrotiri in particolare v. Karali, 1990, p. 414; Reese, 1987, p. 205.

26 Su questi 3 centri v. Brysbaert, 2003, in particolare tav. XliIb; Brysbaert, 2007, p. 32, tav. 1.

27 Per Hala Sultan Tekke v. Brysbart, 2007, p. 32, tav. 1; Reese, 1987, p. 205; per Sarepta v. Brysbaert, 2007, p. 32, tav. 1; Reese, 1987, p. 205; per Lachish e Ascalona v. Bar-Yosef Mayer, 2005, p. 48; per Tell el-Dab’a v. Brysbaert, 2002, pp. 104-105.

28 BRYSBAERT, 2002. 
Tab. 1 - Attestazioni archeologiche del riuso intenzionale di conchiglie a fini costruttivi.

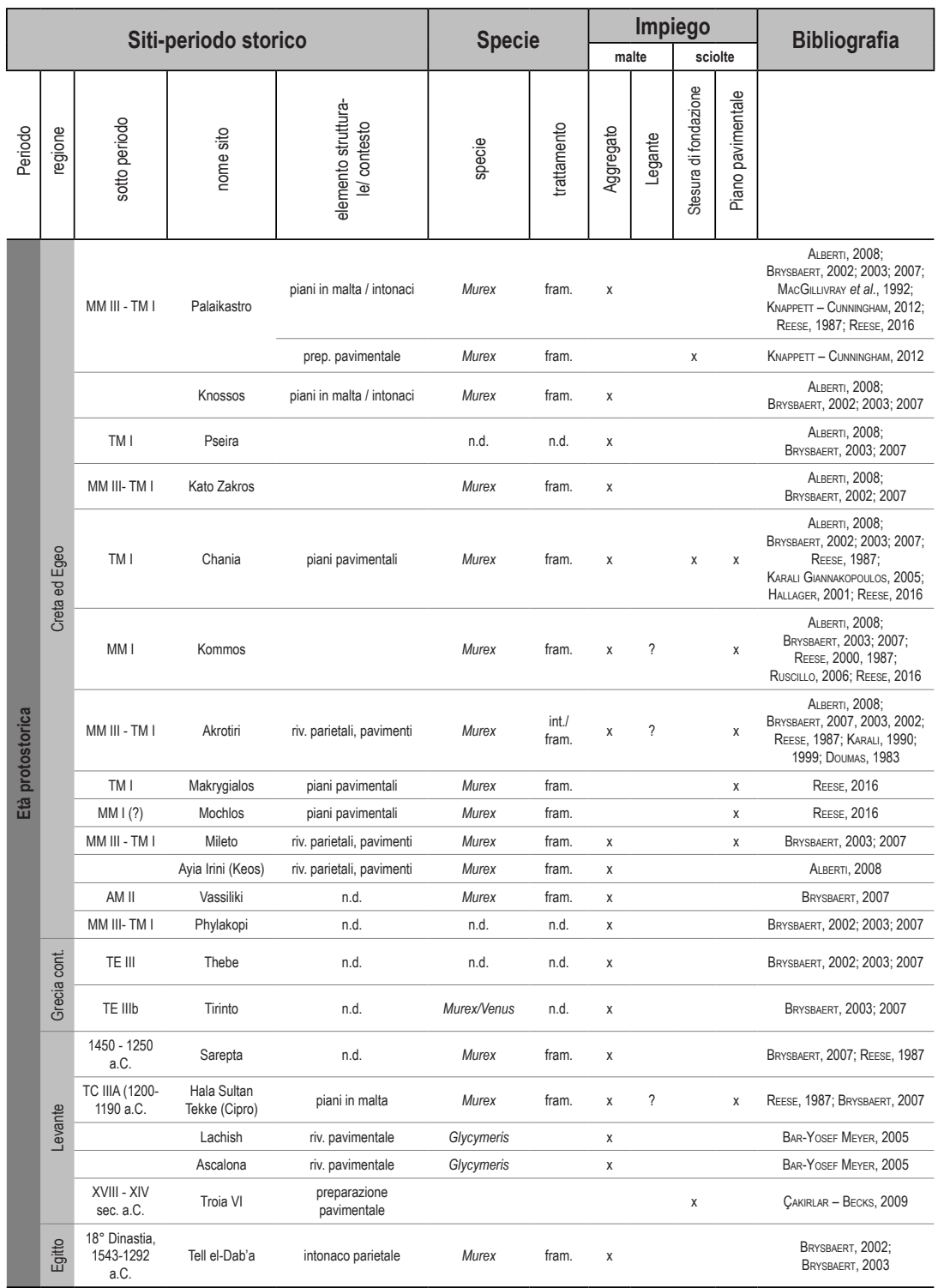




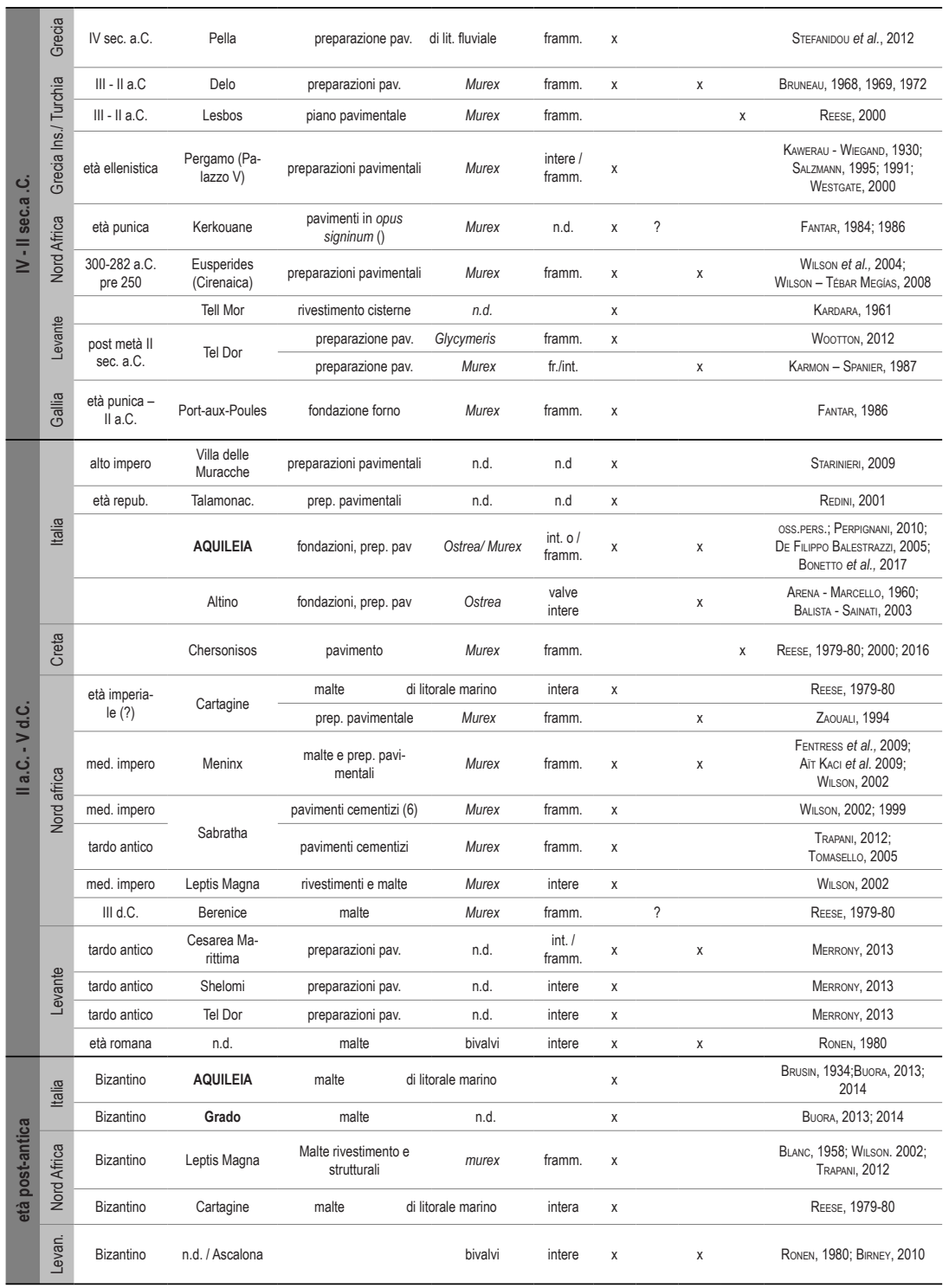

Mancano sostanziali riferimenti relativi all'età arcaico-classica, mentre dall'età greco-ellenistica la presenza di conchiglie, sempre delle specie del murex, è documentata in particolare nelle preparazioni dei pavimenti, soprattutto musivi; anche in questo caso le attestazioni provengono da siti dove è supposta essere praticata la produzione della porpora. L'isola di Delo rappresenta il caso emblematico per questo periodo: oltre alla documentazione archeologica che dimostra una sicura 
produzione della preziosa tintura ${ }^{29}$, i residui conchigliferi di tale attività venivano spesso impiegati nelle malte di supporto di mosaici ${ }^{30} \mathrm{o}$ in stesure nei sottofondi di preparazione ${ }^{31}$.

Altri casi documentati sono a Pergamo ${ }^{32}$ e Pella $^{33}$, seppur in quest'ultimo caso la sporadica presenza di conchiglie di specie non precisate sembrerebbe essere dovuta all'impiego di sabbie fluviali non setacciate ${ }^{34}$.

Da questo periodo le attestazioni di presenze di gasteropodi o bivalvi nelle malte provengono anche dal Mediterraneo occidentale soprattutto in area nordafricana e ancora una volta in centri dove era attiva l'industria della porpora. Murici nelle malte sono infatti presenti a Kerkouane $^{35}$ e a Eusperides (Fig. 1) ${ }^{36}$. Sulla costa levantina attestazioni provengono dalla preparazione di un mosaico in vermiculatum di sicura matrice ellenica a Tel Dor ${ }^{37}$, e dalle malte strutturali e di rivestimento di cisterne a Tel Mor $^{38}$. Ancora, a Port-aux-Poules, in Gallia Meridionale, sono presenti murex frammentati nelle malte di un forno di età punica ${ }^{39}$.

La tecnica continua ad essere attestata nella successiva età romana prevalentemente nelle medesime aree geografiche dove tali prassi erano adottate anche in passato. Dal nord Africa, gusci di murex sono reimpiegati nelle malte strutturali e di rivestimento a Meninx (Djerba) ${ }^{40}$ e Car-

29 Bruneau, 1969, pp. 767 ss.; Bruneau, 1978.

30 Attestazioni di reimpieghi abbondanti di gusci di murice nelle malte provengono da un piano pavimentale presso la Casa I C del Quartiere delle Stadio in associazione con frammenti di marmo e ceramici, dalle malte preparatorie di un mosaico rinvenuto a sud della sinagoga (v. BRUNEAU, 1969, pp. 766-767, n 5; BRUNEAU, 1972, p. 21, n. 112) e nelle malte di emblemata pavimentali (BRUNEAU, 1972, p. 208, n. 160, fig. 129 e p. 230 , n. 202).

31 Su quest'ultimo aspetto cfr. infra.

32 Murex trunculus, interi, trovati nella malta di un pavimento in mosaico. v. Kawerau - Wiegand, 1930, pp. 53-55 e fig. 70; Salzmann, 1995, pp. 106-107, pl. 16.1; SALZMANN, 1991, p. 436.

33 Stefanidou et al., 2012, p. 740, tav. 1 e fig. 2a, p. 742.

34 Stefanidou et al., 2012, p. 749.

35 A Kerkouane sono spesso impiegate malte con tritume conchigliare (murici) nei rivestimenti e piani pavimentali (FANTAR, 1984, p. 497, nota 200; FANTAR, 1986, p. 509). Viene in particolare segnalata la presenza di abbondanti conchiglie fracassate nelle malte di un pavimento in opus signinum presso la porta Nord (v. FANTAR, 1984, p. 497, nota 200).

36 Murici fracassati sono presenti in un pavimento di V sec. a.C. dell'area Q degli scavi della Società di Studi libici (WiLson - TéBar Megías, 2008, p. 232), e nella preparazione di un mosaico a ciottoli dell'area $\mathrm{P}$ databile alla prima metà del III sec. a.C. (Wilson - TÉbar Megías, 2008, p. 233 e fig. 6; Wilson et al., 2004, p. 156).

37 Wоoтton, 2012, p. 221; tuttavia la presenza della specie Glycymeris, tipica di litorali marini, dimostra l'impiego di sabbie non lavate ed un uso forse non intenzionale.

38 Kardara, 1961, p. 263. Non viene specificata la specie di conchiglia.

39 FANTAR, 1986, pp. 508-509.

40 Wilson, 2002, p. 430; 2004, p. 161. Sono particolarmente frequenti murici in malte di rivestimento di cisterne e vasche (v. Fentress et al., 2009, p. 170; Drine, 2008, pp. 130-132; Wilson, 2004, pp. 160-161); murici sono infatti impiegati nelle malte di 


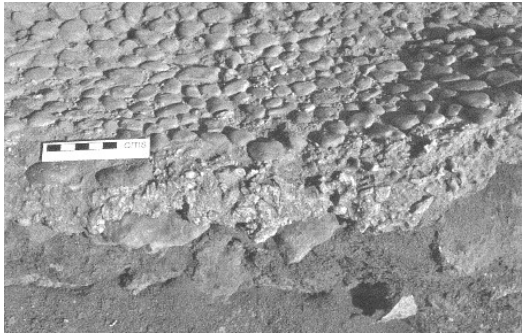

Fig. 1 - Berenice (Eusperides). Mosaico a ciottoli con preparazione in malta ricca di murici, con funzione di aggregato (Da WiLson - TÉBAR MEGías, 2008, p. 233, fig. 6).

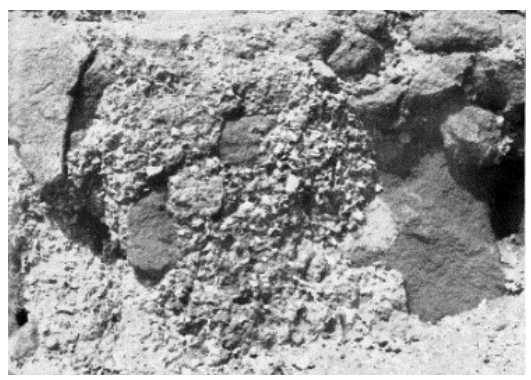

Fig. 3 - Leptis Magna. Malta di murici nelle mura bizantine della città (Da BLANC, 1958, pl. XCIV).

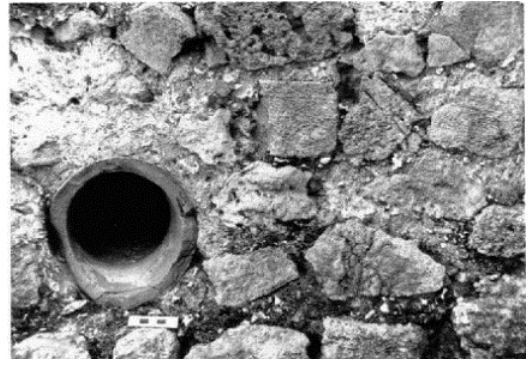

Fig. 2 - Leptis Magna. Gusci di murice nelle malte di allettamento del Chalcidicum (Da WILSON, 2002, tav. IV, 1).

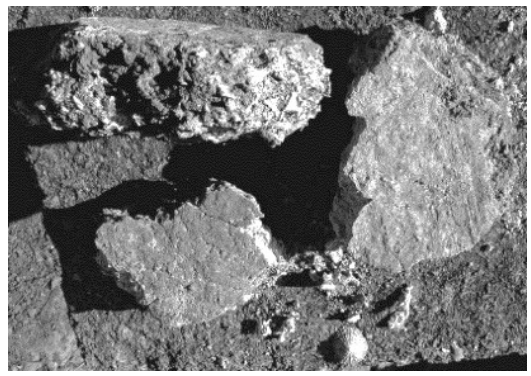

Fig. 4 - Leptis Magna. Frammenti di pavimenti cementizi con frammenti di murice (Da TRAPANI, 2012, p. 223, fig. 11).

tagine $^{41}$, nei supporti di pavimenti e rivestimenti cementizi a Sabratha di età romana medio imperiale e tardo antica ${ }^{42}$ e, soprattutto, a Leptis Magna dove gusci di murice sono impiegati nelle malte dei giunti murari delle cisterne del Chalcidicum (databili tra 120 d.C. e l'inizio del III sec. d.C., Fig. 2) e nelle malte di rivestimento di cisterne collocate ad est delle latrine presso le Terme di Adriano ${ }^{43}$.

pavimentazioni di un peristilio di un'abitazione romana (v. Аїт КАCI et alii, 2009, p. 219), nei rivestimenti parietali e pavimentali di alcuni ambienti degli horrea e di cisterne (v. Aïт KACI et alii, 2009, pp. 224-226), nonché nei rivestimenti di acquedotti (v. Aïт KACI et alii, 2009, p. 234). Tutti i contesti sono riferibili a fasi romano imperiali o tardo antiche.

41 ReEse, 1979-80, p. 90 e fig. 7, p. 92. La pratica continua anche nella successiva età bizantina. Le conchiglie in questo caso sono bivalvi di ambiente peri-costiero.

42 I gusci di murice costituiscono l'unico aggregato nelle malte di 4 pavimenti in cementizio di età medio imperiale, mentre in altri due sono impiegati in associazione a frammenti fittili (v. Wilson, 2002, p. 433; WiLson, 1999, pp. 42-44 e figg. 16-17, p. 43). Pavimenti analoghi sono si trovano in contesti tardi nella Casa di Liber Pater e nella c.d. "Casa della Piscina" (Trapani, 2012, p. 226, nota 49).

43 Wilson, 2002, pp. 433-435 e tav. IV, figg. 1-2; la realizzazione delle cisterne ad est delle latrine delle Terme di Adriano, certamente successiva al 120 d.C., viene da 
Maggiori attestazioni provengono dalla successiva età bizantina, dove tali impasti sono impiegati nelle volte dei serbatoi del ninfeo ad ovest del Chalcidicum ${ }^{44}$, nei giunti e nel nucleo strutturale dell'apparecchiatura muraria della cinta giustinianea di difensa della città (Fig. 3 $)^{45}$, nella Basilica del Foro vecchio e antistante battistero e nella realizzazione di una piccola fontana lungo il Kardo massimo; infine alcuni pavimenti cementizi del frantoio presso il Ninfeo dell'Eracle nei quartieri "suburbani" della città sono ugualmente realizzati mediante l'impiego di malta con abbondanti murici (Fig. 4$)^{46}$.

Dalla costa orientale attestazioni per il periodo tardo antico vengono da Cesarea Marittima, dove conchiglie sono spesso impiegate nelle preparazioni di alcuni mosaici (spesso in associazione con altri materiali) e in piani in malta (tardoantichi?) da Shelomi e Tel Dor ${ }^{47}$. Viene inoltre fatto presente da A. Ronen come l'uso di conchiglie, in particolare di specie bivalvi, come aggregato nelle malte sia frequentemente attestato in diversi siti della costa levantina di età romana, bizantina ed oltre ${ }^{48}$.

Per quanto riguarda l'area italica le attestazioni per l'età romana non sembrano essere così frequenti e sono raramente riportate dagli autori. Nei casi di seguito citati non sono chiare né la tipologia né la frequenza in presenza di conchiglie nelle malte. Ciò non permettere di comprendere se il loro impiego fosse intenzionale o meno. Attestazioni provengono prevalentemente dalle preparazioni di pavimenti musivi in siti prossimi alle coste, come nella c.d. villa delle Muracche a Tortoreto ${ }^{49}$ e a Talamonaccio (Orbetello) $)^{50}$.

In Italia settentrionale i riferimenti ad impieghi di conchiglie in malte non sembra essere frequente in letteratura. Ne è riportata la presenza da M. Buora nei giunti delle murature del Battistero di Elia a Grado, databile al VI sec. d.C., nonché nelle mura bizantine di Aquileia ${ }^{51}$.

alcuni fatta risalire alla piena età bizantina (Tomasello, 2005, p. 151; TrAPANI, 2012, p. 221).

44 Tomasello, 2005, pp. 145, 150-151; Trapani, 2012, p. 221.

45 BlanC, 1958, p. 187, pl. XCIV-XCV, citato da Wilson, 2002, p. 433.

46 Trapani, 2012, pp. 218, 222 e fig. 11, p. 223.

47 Per Cesarea Marittima v. Merrony, 2013, pp. 67-69, in part. p. 69; per Shelomi e Tel Dor v. Merrony, 2013, p. 69, nota 34. Essendo rinvenute in associazione con altri materiali e non essendo definita la frequenza in presenza non è possibile valutarne con certezza l'intenzionalità di impiego.

48 Ronen, 1980, p. 170; ad esempio per Ascalona cfr. Birney, 2010.

49 STARINIERI, 2009, pp. 62-72. Rinvenute conchiglie in quasi tutte le malte preparatorie dei pavimenti della villa.

50 Redini, 2001, p. 231. Le conchiglie sono qui impiegate nel nucleus (sp. $3 \mathrm{~cm}$ ) di un mosaico pavimentale in associazione a pietrisco minuto.

51 Buora, 2013, p. 569; Buora, 2014, p. 12. Per Aquileia cfr. infra. 


\section{PROPRIETÀ E IMPIEGO DI CONCHIGLIE COME PREPARAZIONE O FONDAZIONE}

È già stato osservato come conchiglie utilizzate in strati di fondazione o preparazione di piani pavimentali possano fungere da ottimo strumento di bonifica; solide ma al contempo leggere, permettono un adeguato isolamento dall'umidità del sottosuolo e forniscono stabilità alle fondazioni di strutture murarie, pavimenti e strade ${ }^{52}$. Ancora ai giorni nostri, in alcune zone di area levantina, gli artigiani locali esaltano le prestazioni delle specie bivalvi nella costruzione di preparazioni pavimentali ${ }^{53}$. Ma anche in antico si dovevano possedere conoscenze empiriche della fisica dei terreni e dellimportanza del corretto drenaggio delle acque sotterranee, al fine di prevenire umidità ed allagamenti ${ }^{54}$. Spostandosi all'ambito agricolo, lo stesso Virgilio, nel I sec. a.C. (Georg., II, 348-350), ricorda infatti come sia fondamentale disporre pietre porose o conchiglie sotto terra in occasione della semina dei virgulti delle viti al fine di mantenere il suolo ben areato e drenato (aut lapidem bibulum aut squalentis infode conchas; inter enim labentur aquae, tenuisque subibit halitus atque animos tollent sata $)^{55}$. Il passo viene citato un secolo dopo da Columella (De agr., III, 15, 3-4), dove si ricorda come lo stesso Magone, letterato cartaginese, richiamasse l'importanza del drenaggio del terreno, seppur il primo a menzionare il ruolo specifico delle conchiglie a tal fine sembrerebbe essere l'autore latino ${ }^{56}$.

Tuttavia, ancora una volta, dai riferimenti in letteratura non è facile distinguere l'impiego intenzionale di conchiglie in strati a composizione omogenea e regolarmente apprestati dalla loro disorganica occorrenza in eterogenei riporti terrosi posti con funzione di rialzo o bonifica al di sotto di piani pavimentali o strutture. Tale distinzione è nondimeno fondamentale per comprendere se sia stata operata una precisa scelta nell'impiego di conchiglie come materiale per vespai e, per diretta conseguenza, se le maestranze conoscessero le proprietà di tali materiali in antico.

52 Ronen, 1980, p. 171 citato da Bar-Yosef Mayer, 2005, p. 48; Law, 2014, p. 246; CECI, 1984, pp. 67-68.

53 Ronen, 1980, p. 171.

54 Antico Gallina, 2011, p. 4.

55 Nella versione critica di R.A.B. Mynors (Mynors, 1990, p. 143) si suppone che tale pratica debba derivare necessariamente da conoscenze maturate in ambito greco, in quanto in Italia non sono presenti vitigni di età romana collocati presso le coste, cioè nei luoghi in cui è più facile avere acceso a depositi conchigliferi. Tuttavia i principali centri viticoli di età romana del nord Italia sono collocati proprio presso la costa, nel territorio di Altino e Concordia (Di Filippo Balestrazzi, 2004).

56 Teofrasto (Cause dei fenomeni vegetali, III, 4, 3), riguardo la medesima pratica legata alla semina, cita la preparazione di una fossa in terra da riempire con sole pietre, mentre non vi è alcuna menzione alle conchiglie. Anche Catone (De agr., L-LI, 43), non riporta tale prassi. 
Verranno in seguito discussi i casi in cui sia documentato l'impiego intenzionale di conchiglie in apprestamenti di fondazione o preparazione pavimentale (Tab. 1).

\section{Attestazioni nel Mediterraneo}

Tralasciando qui i casi in cui conchiglie furono impiegate come materiale compattante di rivestimenti pavimentali a matrice terrosa ("floor packing") - pratica attestata in diversi siti di area egea e a Creta in fasi medio e tardo minoiche ${ }^{57}$ - attestazioni dell'uso di conchiglie sciolte come livelli di sottofondazione sono meno frequenti rispetto al loro impiego nelle malte. Ciò può essere dovuto, come si diceva, alla difficoltà di distinguere stratificazioni di conchiglie appositamente apprestate da più comuni scarichi di macerie comprendenti, tra gli altri materiali, anche malacofauna.

Le più antiche attestazioni provengono, anche in questo caso, dall'area egea. Nelle fasi VI tarda e VIIa di Troia (VI: 1700 - 1300 a.C. o 1250 a.C.; VIIa: 1300 - 1170 a.C.) stesure di soli frammenti di murex sono state trovate in sequenze stratigrafiche nella fondazione di un forno e sotto i piani pavimentali di alcuni ambienti ${ }^{58}$, così come a Palaikastro e Chania (Creta) in fasi medio e tardo minoiche ${ }^{59}$.

Successivamente la tecnica sembra perdurare: a Delo l'impiego di frammenti di murice è chiaramente osservabile tra due pavimenti in mosaico sovrapposti databili al periodo ellenistico (Fig. 5$)^{60}$. Allo stesso orizzonte cronologico è riferibile uno strato di centinaia di frammenti di murice (prob. Hexaplex) rinvenuti tra due pavimenti musivi a Tel Dor ${ }^{61}$. In età romano-imperiale ne vediamo massiccia applicazione nelle fondazioni delle pavimentazioni dei contesti abitativi di Meninx - Djerba

57 Per Akrotiri v. Brysbaert, 2002, p. 104; Doumas, 1983, p. 117; Karali, 1990, p. 414; Karali, 1999, p. 43; Reese, 1987, p. 205; per Mileto v. Brysbaert, 2002, p. 104; per Kommos v. Reese, 2000, pp. 626, 645, pl. 6.11; Ruscillo, 2006, pp. 789, 803, 807809; per Chania v. Hallager, 2001, p. 117 e nota 20; per Hala Sultan Tekke v. Reese, 1987, p. 205; per Makrygialos v. Reese, 2016, p. 410; per Mochlos v. Reese, 2016, pp. 411-412. Più tardi la pratica è attestata a Lesbo in pavimenti di III - II sec. a.C. (v. ReESE, 2000 , p. 645) e in età romana a Limani (Chersonisos) v. ReESE, 1979-80, p. 86; ReESE, 2000, p. 645; REESE, 2016, p. 409.

58 ÇAKIRLAR - BECKs, 2009, p. 95. Le preparazione in particolare del forno consiste in "a thin layer of clay, a layer of sherds, again a clay-layer ca. 3-4 cm thick, then a 1-2 cm thick layer of crushed shells and finally another 3-4 cm thick layer of clay".

59 A Palaikastro diversi pavimenti in malta databili prevalentemente al MM II erano collocati sopra a letti di frammenti di murice fracassati (KNAPPETT - CUNNINGHAM, 2012, pp. 10, 66); per Chania la preparazione in murici si data al TM I cfr. KARALi Giannakopoulos, 2005, p. 162.

60 Bruneau, 1968, p. 675, nota 1; Bruneau, 1969, p. 766, n³ e fig. 3, p. 767; BruNEAU, 1972, p. 124, n. cat 7 e fig. 10, p. 120.

61 Karmon - Spanier, 1987, p. 155, fig. 11. 


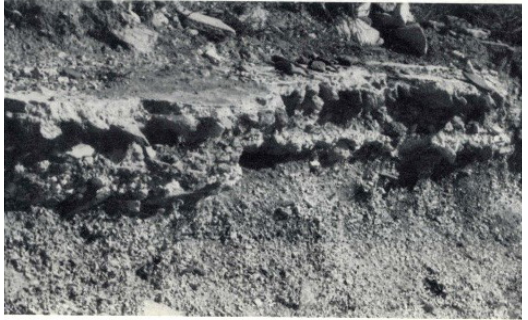

Fig. 5 - Delo. Sottofondo preparatorio di pavimento musivo realizzato con soli frammenti di murice (Da BRUNEAU, 1972, p. 120, fig. 10).

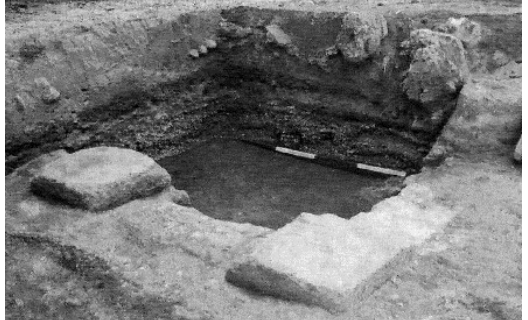

Fig. 6 - Meninx (Djerba). Sottofondo preparatorio di pavimento di abitazione realizzato con soli frammenti di murice ( $\mathrm{Da}$ FENTRESS et al., 2009, p. 163, fig. 10.38).

(I-II sec. d.C., Fig. 6) ${ }^{62}$ e di Cartagine ${ }^{63}$. In entrambi i casi si tratta, ancora una volta, di gusci di murex.

Per l'età tardo antica attestazioni provengono da Cesarea Marittima, dove conchiglie sono usate come vespai di alcuni mosaici ${ }^{64}$ mentre in età bizantina sono ancora impiegate nelle fondazioni di diversi piani pavimentali in area levantina ${ }^{65}$.

Per quanto riguarda l'Italia settentrionale, l'uso di conchiglie nell'apprestamento di fondazioni e preparazioni, non solo di pavimenti, è attestata anche oltre i casi aquileiesi. Ad Altino, in particolare, viene riportato negli anni ' 60 da A. Marcello e M. Arena il rinvenimento, al di sopra dell'argilla naturale, di due depositi di $6 \times 1 \mathrm{~m}$ e 4,5x3,5 m composti esclusivamente da valve d'ostrica. Nel primo caso le valve sono disposte in uno strato di spessore di $15 \mathrm{~cm}$ e sono ricoperte da un livello di segatura spesso circa $40 \mathrm{~cm}$, al di sotto di un terreno con frammenti fittili (vespaio?), a sua volta posto a preparazione di un pavimento in cotto. Nel secondo caso, al di sopra dell'argilla sterile, è presente un primo strato di ostriche e frammenti fittili $(\mathrm{sp} .50 \mathrm{~cm} \mathrm{ca})$, segue uno strato di sole ostriche (sp. $20 \mathrm{~cm}$ ), coperte da livello terroso con macerie fittili. Non viene qui individuata traccia della pavimentazione in cotto $^{66}$. La notizia viene in seguito segnalata ${ }^{67}$, seppur l'interpretazione degli strati con

62 Attestato nei riporti di fondazione di strutture di età romana comprese tra I e II sec. d.C. in diverse abitazioni (Aїт KACI et al., 2009, p. 215 e Fentress et al., 2009, p. 166 e fig. 10.38, p. 163), di un peristilio scoperto (Аїт КАCı et al., 2009, p. 219) e nei riporti di preparazione di acquedotti a terra (Аїт KАС et al., 2009, p. 234).

63 Il più antico di questi depositi si data a cavallo tra I sec. a.C. e I sec. d.C. (ZAOUALI, 1994, pp. 322-323).

64 Merrony, 2013, pp. 67-68. Essendo usate in associazione con altri materiali quali frammenti fittili o pietre, non è possibile valutarne la densità totale e l'intenzionalità nell'uso.

65 Ronen, 1980; Ad esempio per Ascalona cfr. Birney, 2010.

66 Arena - Marcello, 1960, p. 119.

67 Balista - SAinati, 2003, p. 333. La descrizione dei due contesti è qui leggermente differente, in quanto viene affermato che sono entrambi coperti da segatura (rin- 
conchiglie a funzione di preparazione del pavimento non sia mai stata chiaramente sostenuta ${ }^{68}$. Sempre ad Altino, un caso certo di reimpiego di conchiglie a fine edilizio è nella stesura di ostriche, in associazione a frammenti fittili, impiegata come fondazione della "porta approdo" della città, databile tra il II e la prima metà del I sec. a.C. ${ }^{69}$.

In Istria sono inoltre stati rinvenuti diversi depositi con murici fracassati al di sotto di pavimenti che, seppur non sembrino essere disposti in "stesure ordinate" ma piuttosto mescolati in riporti terrosi, vengono comunque supposti essere intenzionalmente impiegati al fine di migliorare l'isolamento dei piani soprastanti ${ }^{70}$.

\section{CASI AOUUILEIESI}

Le attività di scavo condotte dal 2007 ad Aquileia dall'Università degli studi di Padova in due contesti abitativi di grande rilevanza, la cosiddetta Domus di Tito Macro ai Fondi Cossar e la Casa delle Bestie Ferite, si sono rivolte allo studio dellevoluzione architettonica di queste ricche residenze private nel corso del tempo ${ }^{71}$. Dagli scavi sono venuti in luce tre contesti stratigrafici, pertinenti a fasi differenti, dove considerevoli quantità di gusci di mollusco sono state impiegate come elemento da costruzione.

\section{Caso 1}

Al di sotto di un mosaico nella Casa delle Bestie Ferite, pesantemente spoliato e preliminarmente databile attorno alla fine del IV sec. d.C. ${ }^{72}$, è stato rinvenuto un consistente numero di conchiglie, per lo più valve di ostrica intere del tipo Ostrea Edulis e Ostrea sp., e sporadici Cerastoderma $s p .^{73}$, all'interno della malta del massello preparatorio del pavimento, in associazione ad abbondanti paste vitree e frammenti vitrei, ceramici e di intonaco (Fig. 7) ${ }^{74}$. La matrice della malta di calce era biancastra, particolarmente friabile e facile a disgregarsi.

\section{Caso 2}

venuta esclusivamente nel primo deposito) e sono contenuti "all'interno di due fosse" non espressamente dichiarate nel testo originario.

68 Viene affermato in Marzano, 2013, p. 192 che "it cannot be excluded that the sawdust layer [strato di segatura n.d.a.] had something to do with the building of the floor at a later time".

69 Balista - Sainati, 2003, p. 333.

70 Macheboeuf, 2013, in particolare p. 269.

71 Bonetto - Salvadori, 2012; Bueno - Centola, 2014; Bueno et al., 2014.

72 Datazione basata sul rinvenimento di frammenti ceramici il qui terminus post quem è riferibile a questo periodo (V. Mantovani, comm. pers).

73 Si ringrazia la dott.ssa Martina de March per l'analisi dei reperti e il riconoscimento delle specie.

74 US 1465. Boschetti et al., 2016, pp. 21-22; Dilaria, 2014, scheda Aq CBF Prep 28. 


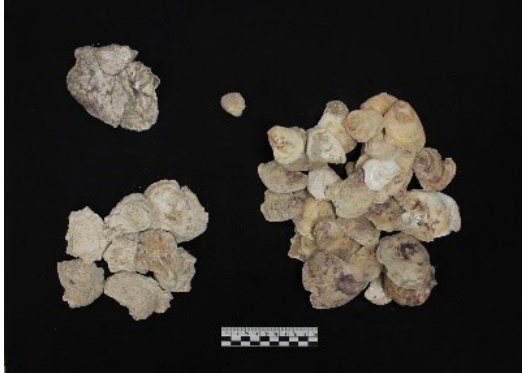

Fig. 7 - Gusci di mollusco (ostrea sp., cerastoderma, ostrea edulis), nelle malte.

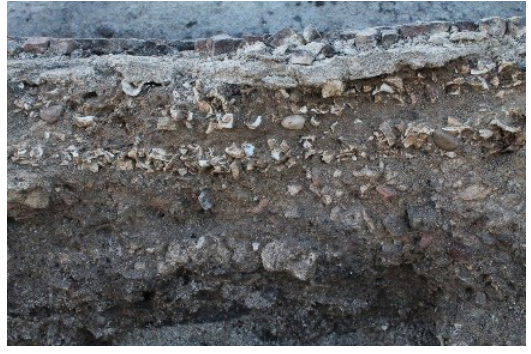

Fig. 8 - Strato di murici in frammenti al di sotto di un mosaico di IV sec. d.C. presso la Domus delle Bestie ferite (foto autore).

Un tessellato geometrico policromo con squame nella domus delle Bestie ferite, adiacente al mosaico precedente trattato, ed inquadrabile nella prima metà del IV sec. d.C. ${ }^{75}$, presenta, al di sotto del massello preparatorio ed in parte nell'interfaccia con la stessa malta di allettamento del pavimento, una stesura continua di conchiglie (spessore $6-10 \mathrm{~cm}$ circa), formata quasi esclusivamente da gusci di gasteropodi altamente frammentari, probabilmente del tipo Hexaplex Trunculus o Bolinus Brandaris, seppur non sia risultata facile l'identificazione della specie a causa dell'elevato stato di frammentazione in cui vertono (Fig. 8) ${ }^{76}$.

\section{Caso 3}

All'interno di una struttura circolare, rinvenuta presso l'area delle botteghe contigue alla domus di Tito Macro e preliminarmente interpretata come forno per alimenti databile al I sec. d.C., è stato rinvenuto un livello costituito interamente da 911 conchiglie, disposte in una stesura uniforme alla quota della fondazione della camera di cottura, al di sotto di una serie di apporti argillosi funzionali alla creazione di piani da fuoco (Fig. 9) ${ }^{77}$. Le specie afferiscono prevalentemente a gasteropodi $(\mathrm{mu}-$ rex sp. 733), o bivalvi (ostrea sp. 175), mentre 3 esemplari restano indeterminati ${ }^{78}$. Le conchiglie sono quasi sempre intere, e le valve spaiate.

A questi casi se ne aggiungono altri, sempre da Aquileia, riportati da studiosi negli anni passati.

\section{Caso 4}

Ancora presso l'area dei Fondi Cossar, dove sono presenti alcune domus signorili di epoca romana, viene segnalata nei diari di L. Bertac-

75 Bueno et al., 2010, pp. 1-15.

76 Strato non numerato; mosaico: US 8. v. Dilaria, 2014, scheda Aq CBF Prep 16.

77 US 7232=7358. v. Centola, 2013, pp. 31-35; Centola comm. pers.

78 Tutte le conchiglie della stesura sono state recuperate ed analizzate. Si ringrazia la dott.ssa Martina de March per l'analisi dei reperti e il riconoscimento delle specie. 


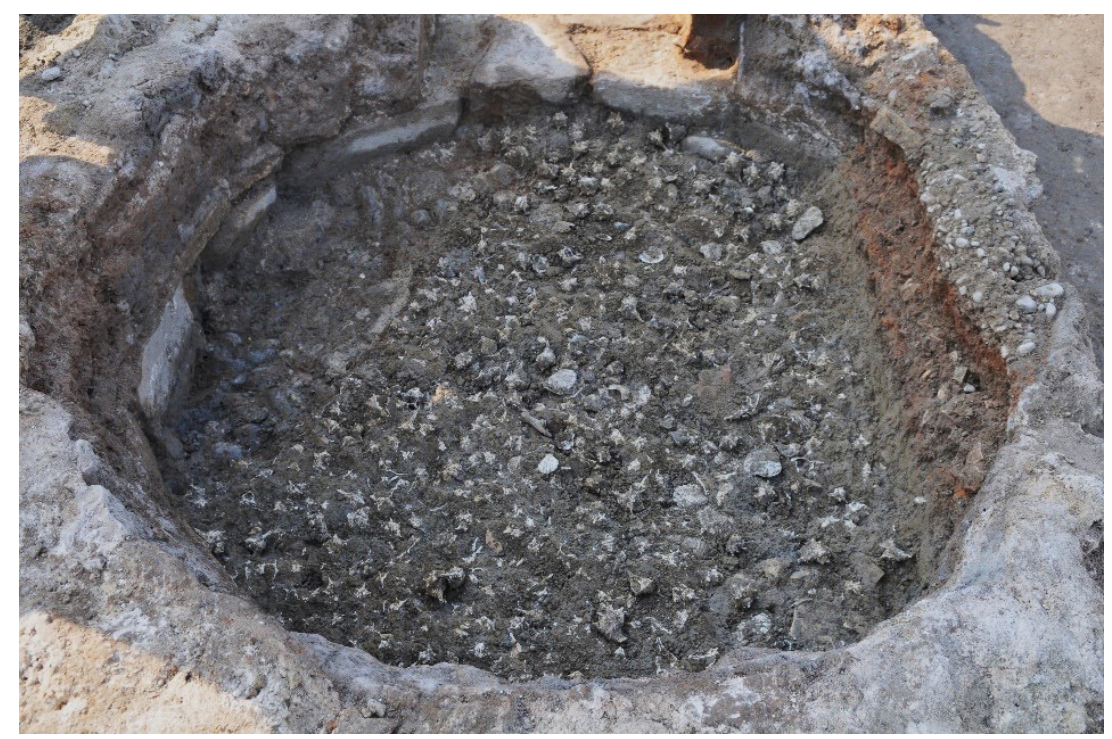

Fig. 9 - Aquileia - Strato di ostriche e gasteropodi nella fondazione del forno presso le botteghe adiacenti alla Domus di Tito Macro (foto V. Centola).

chi la presenza di un mosaico con preparazione in malta di $10 \mathrm{~cm}$ composta di sola calce e numerosissime conchiglie (murici interi). Il vespaio del pavimento era invece realizzato con ciottoli sciolti ${ }^{79}$.

\section{Caso 5}

Vi è attestazione della presenza di conchiglie macinate nelle malte dell'emblema dell'c.d. Asaroton oikos, tradizionalmente datato tra la fine del II secolo e l'inizio del I sec. a.C., rinvenuto ancora nell'area dei Fondi Cossar e attualmente conservato presso il Museo Archeologico Nazionale di Aquileia ${ }^{80}$. Tuttavia è probabile che il manufatto sia di importazione, come spesso accade per gli emblemata pavimentali (Fig. 10).

\section{Caso 6}

Viene riportato dal Brusin l'impiego di "malta grassa fatta con sabbia conchiglifera" per la costruzione delle mura bizantine di Aquileia ${ }^{81}$. In seguito ad ispezione autoptica, si è osservato che si tratta di specie diverse di conchiglie di litorale costiero, impiegate abbondantemente, sia intere che finemente tritate, come aggregato nei composti (Figg. 11-12).

79 Bonetto et al., 2017, p. 48; Di Filippo Balestrazzi, 2005, pp. 200-201.

80 Perpignani - Fiori, 2012, pp. 119, 125.

81 BRUsin, 1934, pp. 47-48; La presenza di conchiglie nelle malte non può essere considerata comunque un dato a favore della datazione bizantina della struttura, come proposto da M. Buora (Buora, 2013, p. 567; Buora, 2014, p. 12). 


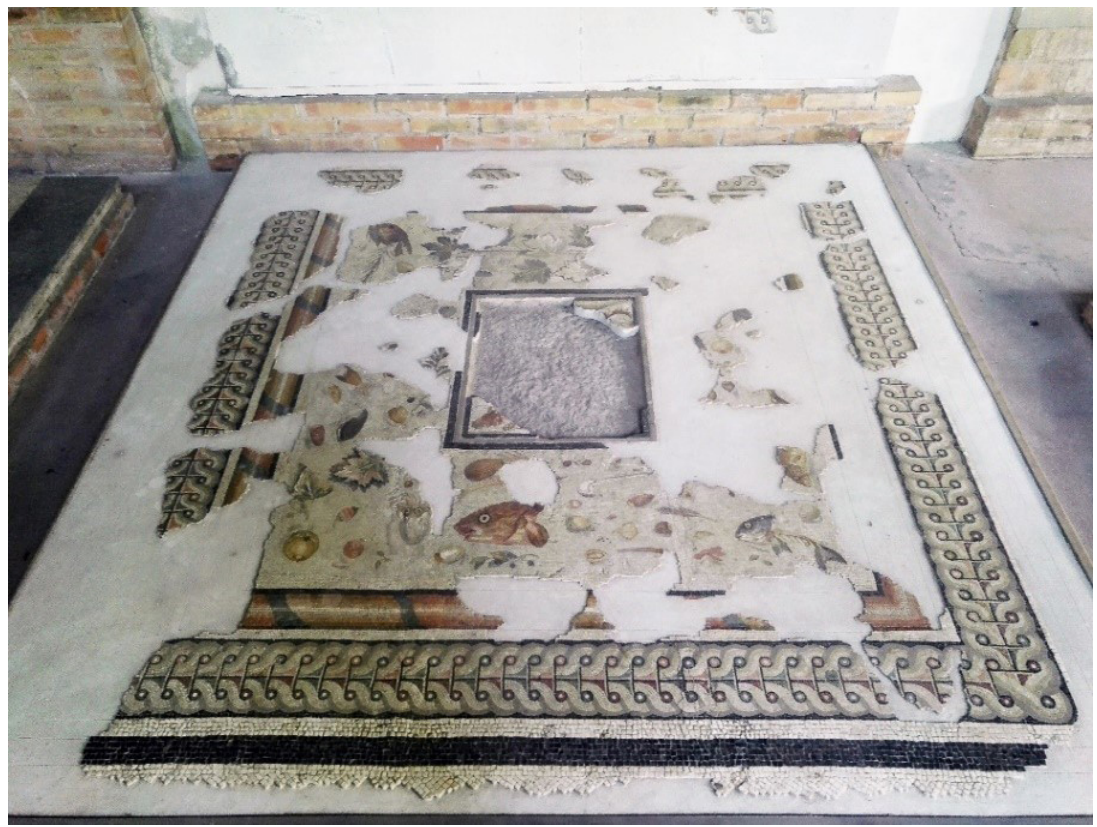

Fig. 10 - Asaroton oikos. Museo Archeologico Nazionale di Aquileia (foto autore).

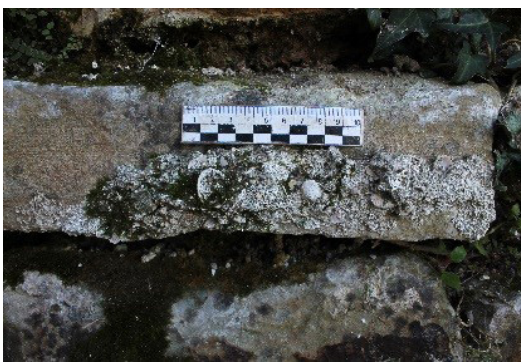

Fig. 11 - Conchiglie di litorale marittimo (intere e in frammenti) nelle malte delle mura bizantine di Aquileia (foto autore).

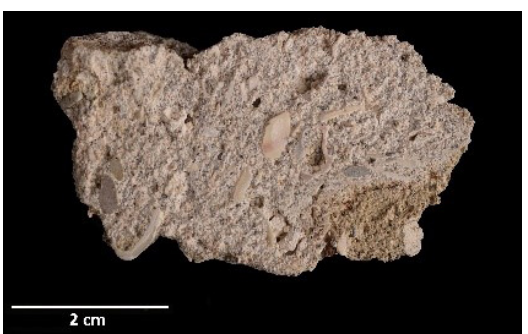

Fig. 12 - Sezione petrografica di un campione di malta di giunto murario prelevato dalle mura bizantine di Aquileia (foto autore).

\section{CONCLUSIONI. L'IMPIEGO DI CONCHIGLIE COME MATERIALE DA} COSTRUZIONE AD AOUILEIA.

Attraverso l'analisi dei contesti in esame ed il confronto con altri siti, seppur non esaustivo per le singole aree geografiche e i periodi storici qui presi in esame, si possono trarre alcune considerazioni, sintetizzabili attorno a due questioni cruciali, riguardo le modalità e motivazioni che spinsero ad impiegare conchiglie a fini edilizi ad Aquileia:

- Sfruttamento originario, intenzionalità d'uso e funzione delle conchiglie come materiale edilizio; 
- Diffusione della pratica e trasmissione della specifica tradizione costruttiva.

\section{Sfruttamento originario, intenzionalità d'uso e funzione delle conchig- lie come materiale edilizio}

Per quanto concerne i contesti aquileiesi in esame, gran parte dei casi rimandano a forme di "uso secondario" di conchiglie: nei casi 1 e 3 , a cui si può aggiungere probabilmente anche il caso 4, il reimpiego a fine edilizio deriva da un sicuro consumo alimentare di ostriche e di gasteropodi, particolarmente apprezzati in nord Italia ${ }^{82}$. La pressoché totale integrità dei reperti malacologici permette di distinguere i casi 1 e 3 dal caso 2 nel quale, pur non essendo da scartare l'ipotesi che le conchiglie si fossero fratturate in seguito all'abbandono o fossero state intenzionalmente fracassate al momento della messa in opera del pavimento, $i$ gusci di murex vertono in uno stato frammentario del tutto comparabile a quello osservato in altri rinvenimenti per i quali è stato riconosciuto l'esito del processo estrattivo della ghiandola porporifera ${ }^{83}$. Questa nuova notizia di una possibile produzione di tale tintura ad Aquileia è già stata brevemente discussa altrove ${ }^{84}$.

Nello specifico, nel massello preparatorio del mosaico di V sec. d.C. presso la Domus delle Bestie Ferite (caso 1), la presenza di alcune valve dostrica in associazione a diversi intonaci e frammenti in pasta vitrea mescolati nella malta potrebbe essere legata esclusivamente al reimpiego - non intenzionale - di materiali direttamente reperibili in situ, tra cui le conchiglie. Valve d'ostrica caratterizzano infatti pure il sottostante riporto terroso, la cui colorazione scura è da collegare a degrado di sostanza organica in livelli di abbandono in area urbana ${ }^{85}$. Inoltre la modalità di messa in opera non è accurata: le conchiglie si presentano intere e non hanno quindi subito quel trattamento che, come ricorda Palladio,

82 Si rinvengono murici (murex trunculus e brandaris) spesso in associazione ad ostriche negli scarichi antropici provenienti da abitazioni degli scavi a Trieste (Crosada) databili tra I e III sec. d.C. Il fatto che i murici siano rinvenuti interi ha fatto propendere per l'ipotesi di un loro consumo alimentare (Petrucci 2007b; Petrucci, 2008, p. 470). Sempre destinati all'alimentazione erano i murici rinvenuti in diverse contesti ad Aquileia di età repubblicana (Petrucci, 2007a, in part. p. 762).

83 Sul tasso di fratturazione dei murici a seguito dell'estrazione della porpora cfr. a titolo esemplificativo: ÇAKIRlar - Becks, fig. 1, p. 94; CAZzarella et alii, 2005, p. 180, fig. 3; Karali Giannakopoulos, 2005, p. 164, fig. 2; Karali, 1990, p. 414, fig. 4). I murici, laddove non fosse possibile incidere il guscio, potevano essere fracassati, soprattutto se piccoli, per rimuovere la ghiandola porporifera.

84 Dilaria, 2017, in part. p. 5; ulteriori riferimenti alla presenza di depositi di murex ad Aquileia, ritenuti essere esito di tale processo produttivo sono in SCHMIDT, 1893, p. 388; sulle testimonianze di produzione porporaria in Istria v. inoltre MACHEBOEUF et al., 2013.

85 US 1526. Boschetti et al., 2016, p. 22; BRIDI, comm. pers. 
dovrebbero consentire una presa migliore della malta che si presentava infatti, dopo valutazione empirica, estremamente friabile.

L'intenzionalità nell'impiego specifico di conchiglie a fini edilizi è invece chiara per il caso 3, dove queste sono impiegate in uno strato omogeneo e uniformemente distribuito all'interno dell'area del forno realizzato in un momento precedente l'impianto della domus di Tito Macro. Ugualmente, anche la stesura di murici al di sotto del mosaico di IV sec. d.C. della Domus delle Bestie Ferite appare intenzionale, andando a costituire una sorta di composizione alveolata comparabile, sotto il profilo funzionale, allo statumen vitruviano, cioè quel vespaio a funzione isolante da porre al di sotto del massello di preparazione di pavimentazioni di prestigio (mosaici/pavimenti in opus sectile) ${ }^{86}$. La scelta che può aver portato a prediligere, in particolar modo nel caso 3 , le conchiglie rispetto ai più comuni scapoli lapidei o frammenti in cotto nella predisposizione di vespai potrebbe essere derivata anche da una combinazione di più fattori: cioè discreta robustezza e, al contempo, peso specifico contenuto dei gusci. Quest'ultima caratteristica è da tenere in massima considerazione qualora si vada a costruire su terreni come quelli della pianura Padana che, a causa della presenza di falde idrostatiche superficiali, risultano essere umidi e particolarmente cedevoli $^{87}$. Applicazione fortunata che trova confronto, come visto, nel vicino sito di Altino, in cui tali caratteristiche geo-pedologiche risultano essere ancora più esasperate a causa dell'estrema vicinanza all'ambiente lagunare $^{88}$.

Medesimo discorso si può sostenere per quanto riguarda loccorrenza di malacofauna nelle malte del mosaico presso i Fondi Cossar rinvenuto da L. Bertacchi (caso 4), dove i murici, che costituiscono l'unico aggregato o di certo quello di gran lunga prevalente, vennero intenzionalmente impiegati nel massello di fondazione del pavimento probabilmente non per migliorare la coesione della malta: ancora una volta, il loro utilizzo da interi, e non in frammenti, potrebbe essere stato determinato dalla volontà di dotare il supporto di particolare leggerezza per non gravare sul sottostante terreno. Infine, anche per quanto riguarda l'emblema dell'asaroton (caso 5), l'uso di tritume conchiglifero potrebbe essere finalizzato ad alleggerire il supporto, aspetto da tener presente per un manufatto destinato a viaggiare.

Non è chiaro invece se le conchiglie delle mura bizantine (caso 6) siano state aggiunte intenzionalmente o siano da legare semplicemente

86 Vitr., De arch., VII, 1, 1-7.

87 Si veda come per far fronte alla stessa problematica si siano adottate in Cisalpina soluzioni edilizie particolari, che non trovano confronto in area centro italica, come quella delle sottofondazioni pluristratificate di ghiaie drenanti alternate a livelli impermeabilizzanti limo-argillosi (Previato, 2015, pp. 223-231e bibliografia ivi riportata).

88 Mozzi et al., 2011, in particolare pp. 13-14. 
all'uso di grandi quantità di sabbie di litorale costiero non setacciate ${ }^{89}$ in malte e calcestruzzi apprestati senza troppa cura e con una certa frettolosità, per far fronte alla necessità di realizzare una costruzione certamente monumentale ma con tempistiche forse strette, all'indomani della riconquista bizantina dei territori nord-italici ${ }^{90}$. La fitta presenza di conchiglie in frammenti minuti, e quindi forse tritate manualmente, potrebbe cionondimeno rivelare intenzionalità di impiego atta a migliorare la coesione delle malte.

I motivi che spinsero all'impiego volontario di malacofauna a fini edilizi nei casi citati risiedono nella disponibilità e facilità di approvvigionamento in situ della materia prima. Si veda, ad esempio, come soprattutto il forno (caso 3) sia collocato a poca distanza dal porto fluviale di Aquileia ${ }^{91}$, dove è probabile vi fossero mercati o botteghe destinati alla vendita di pesce o di altri prodotti della fauna marittima, i cui scarti, anziché essere gettati nel fiume Natisone ${ }^{92}$, potrebbero essere stati opportunamente riciclati.

\section{Diffusione della pratica e trasmissione di tradizioni costruttive}

Appare innanzitutto chiaro come per qualsiasi periodo storico, in tutto il bacino Mediterraneo, l'impiego più o meno intenzionale di conchiglie a fini edilizi non sembri spostarsi al di fuori di siti collocati in territori peri-lagunari, costieri o insulari. Si può in seconda battuta affermare che le proprietà di questi materiali sopra discusse non fossero ritenute dai costruttori antichi indispensabili a tal punto da creare una domanda ed una conseguente commercializzazione di questo prodotto per l'edilizia. Infatti, l'unico caso in cui conchiglie sono impiegate in contesti strutturali di siti lontani da zone limitrofe a bacini d'acqua proviene dal rinvenimento di murici nelle preparazioni dei mosaici del Palazzo V di Pergamo, aspetto che è apparso particolare pure agli studiosi che ne hanno documentato la presenza ${ }^{93}$. Anche in questo caso non è comunque da scartare l'ipotesi che i murici fossero originariamente giunti in città per motivi differenti (consumo alimentare? ${ }^{94}$ ) e successivamente reimpiegati.

89 A medesime conclusioni è giunto D. S. Reese riguardo la presenza di tali specie nelle malte di strutture romane e bizantine di Cartagine (REESE, 1979-80, p. 90 e fig. 7, p. 92).

90 Sulla cinta bizantina "M4" di Aquileia v. da ultimo Bonetto, 2009, p. 92.

91 Maselli Scotti - Rubinich, 2009, pp. 103-107.

92 In seguito ad un carotaggio effettuato nell'ambito del progetto SARA presso il porto fluviale è stato rinvenuto il paleoalveo del fiume e diversi livelli contenenti molluschi marini in fasi di età romana (MASELli ScotTi et al., 1999, pp. 87-90, fig. 4, p. 85 e in particolare pp. 95-96).

93 KaWERAU - Wiegand, 1930, p. 54. La rocca dista infatti circa $30 \mathrm{~km}$ dal mare.

94 I murici si presentano infatti prevalentemente interi cfr. KAWERAU - WIEGAND, 1930, fig. 70, p. 55. La medesima specie è stata rinvenuta anche in alcune cisterne della 
Per quanto riguarda l'aspetto cronologico-distributivo, si può osservare come le prime attestazioni di impieghi di conchiglie a livello edilizio (età del Bronzo) provengano dall'area egea, per poi allargarsi, nelle epoche successive, anche al resto del Mediterraneo. Il quadro che va così definendosi ricalca abbastanza fedelmente la diffusione della conoscenza della tecnologia legata all'industria della porpora ${ }^{95}$, dalla quale è forse fin troppo condizionato. Come si è detto, il riferimento a presenza/impiego di murex in frammenti è spesso riportato in quanto offre testimonianza della possibile presenza di tale attività artigianale nei centri in cui se ne rinvengono gli scarti conchigliferi. Ė quindi possibile che il reimpiego a fini edilizi di gusci di altre specie di molluschi non venga a tal punto messo in risalto e il dato sia quindi molto più difficilmente reperibile. Si veda ad esempio come nei contesti osservati autopticamente le specie impiegate spesso non siano murici (casi 1, 3, 6) e lo stesso Palladio faccia specificamente riferimento all'uso di gusci d'ostrica nelle malte (cfr. supra). È quindi certo che il quadro delle attestazioni qui riportato sia perfezionabile, ma ciò non vieta di affermare con discreta sicurezza che tali tecniche costruttive fossero state sperimentate solo nei centri in prossimità di litorali costieri sabbiosi e in cui vi fu un forte approvvigionamento di molluschi, tale da richiedere diverse forme di smaltimento dei materiali di scarto: i gusci. Ciò può derivare solo dall'industria della porpora o da un sostenuto consumo alimentare. Come visto, mancano rilevanti attestazioni per l'età arcaico-classica mentre dall'età greco-ellenistica la pratica del riuso di conchiglie a fini edilizi è ancora documentata, seppur sia evidente un calo delle attestazioni in letteratura. Ciò può essere dovuto alla minor attenzione riposta per tali manifestazioni di utilizzo secondario negli studi e report di scavo da parte di coloro che si interessano a temi legati a paleo-dieta e produzione della porpora in età storica, avendo a disposizione testimonianze materiali e fonti letterarie più consistenti rispetto alle epoche precedenti.

Necessario è da ultimo comprendere se le forme di riuso certamente intenzionale di conchiglie a fine edilizio documentate in alcuni dei casi aquileiesi qui presi in esame (casi 2, 3, 4 e forse 5 e 6 ) siano da legare a sperimentazioni a loro modo autonome o piuttosto non rappresentino

\section{città cfr. WootTon, 2012, p. 221 e nota 67.}

95 La maggior parte degli studiosi concordano che le attestazioni più antiche del processo di lavorazione della porpora provengono dall'egeo centrale dell'età del Medio Bronzo e dall'Italia (Coppa Nevigata), e non dal Levante come spesso si crede. Cfr. a riguardo Marzano, 2013, pp. 143-144; Reese, 1987, p. 206; Reese, 2000, p. 643. Tuttavia, non potendo provare l'ipotesi diffusionistica, una scelta rinunciataria sostiene piuttosto che esistettero "molti primi, che osservarono, scoprirono, inventarono, escogitarono, tinsero, produssero, e commerciarono lungo tutto le coste del nostro antico mare" in un arco cronologico comunque circoscritto all'età del Bronzo (v. Alberti, 2006). Sulla questione v. inoltre Karali Giannakopoulos, 2004; Carannante, 2014. 
altro che una delle espressioni periferiche di una koinè tecnica che, alla luce di quanto esposto, vediamo attestata nel Mediterraneo perlomeno dall'Età del Bronzo fino all'età romana ed oltre. Le correlate problematiche che ricadono sotto i concetti di "diffusionismo", "trasmissione culturale" e "trasferimento di tecnologie" sono alcuni dei temi più dibattuti in ambito archeologico e antropologico ${ }^{96}$ e sono così sintetizzabili: è possibile dal dato materiale - in questo caso dall'applicazione di conchiglie a scopi edilizi - ricostruire network commerciali e contatti culturali? O meglio, girando la domanda: dall'osservazione di un medesimo fenomeno in due punti geograficamente distanti e posti cronologicamente in successione si deve sempre premettere un rapporto di trasmissione univoca "da ... a ...", o non è possibile supporre altrimenti che da medesime condizioni storico-sociali ed economico-ambientali due distinti gruppi sociali o compagini etniche possano giungere a medesime soluzioni, tra loro totalmente scisse e a loro modo autentiche, senza necessariamente essere costretti a postulare alcuna forma di influenza di una sullaltra?

Certo è che le pratiche sopra trattate furono sperimentate non solo in antico, ma anche in età moderna ed in culture storicamente tra loro lontane. Si veda ad esempio come le conchiglie siano l'elemento caratterizzante del cosiddetto "tabby", un calcestruzzo prodotto tra 1550 e 1800 nei territori presso le coste sud-orientali statunitensi ${ }^{97}$ e in Centro e Sud America $^{98}$, che reimpiega come sgrassate scarti del consumo alimentare di conchiglie (ostriche in particolar modo), che si rinvengono intere o in frammenti nelle matrici di tali calcestruzzi. L’origine è discussa: non è chiaro se fosse stato portato dagli spagnoli in America o, viceversa, fosse stato importato in Spagna e Portogallo dalla Georgia, in un momento successivo alla conquista ispanica dei territori d'oltreoceano. Ma le attestazioni non si fermano qui. Forse già al tempo dei Maya e ancora nel 900 d.C. nei territori Mesoamericani si utilizzavano conchiglie come aggregato o per la produzione di legante ${ }^{99}$; ancora oggi nel sud dell'India si usano conchiglie marine fratturate come aggregato nelle malte al fine di migliorarne la coesione mentre in Cina conchiglie di terra vengono impiegate per la preparazione di stucchi per intonaci ${ }^{100}$. Anche in Europa,

96 Una sintesi dei diversi orientamenti sul tema dalle teorie darwiniane è in EERKENS - LIPO, 2007.

97 Morris, S. D. “Tabby” New Georgia Encyclopedia. 24 September 2014. http:// www.georgiaencyclopedia.org/articles/history-archaeology/tabby; riportato anche in Reese, 1979-80, p. 90.

98 CECI, 1984, pp. 67-68.

99 Brysbaert, 2007, p. 43; Reese, 1979-80, p. 90. Anche le tecniche legate alla produzione di legante potrebbero essersi diffuse indipendentemente in diverse parti del mondo (v. BrysBaert, 2007, p. 30).

100 Per l'India v. Brysbaert, 2007, p. 43; per la Cina v. Reese, 1979-80, p. 90. Per altri usi e forme di reimpiego moderni v. inoltre CECI, 1984, p. 68. 
alla fine dell'Ottocento, conchiglie erano utilizzate per produrre calce in Olanda e a Gaza sono ancora usate per produrre malte e calcestruzzi ${ }^{101}$.

È quindi possibile supporre che medesime pratiche fossero state sperimentate autonomamente in diverse culture senza per forza presupporre un trasferimento di conoscenze. Tuttavia in un panorama geografico quale il Mediterraneo antico, connotato da uno stretto sistema di network commerciali, è innegabile che vi fossero reciproche influenze declinatesi in molteplici formulazioni "meticcie" sui piani artistico, letterario, tecnologico, sociale, cultuale e culturale.

In tal senso le pratiche qui discusse, dopo le prime sperimentazioni in area egea, sono successivamente documentate soprattutto in territori di cultura greca o dove forte era comunque l'influenza ellenica, come nei territori costieri del nord-Africa e levantini; per questo si potrebbe supporre che pure ad Aquileia le conoscenze su uso e applicazioni a livello costruttivo di conchiglie possano essere state veicolate dallelemento greco attraverso quel canale di scambi e rapporti commerciali privilegiato che fu l'Adriatico ${ }^{102}$. Tale influsso greco-orientale nelle pratiche costruttive è già stato suggerito in passato per quanto riguarda altre manifestazioni edilizie ${ }^{103}$ e sarebbe da ricercarsi nello stanziamento in Cisalpina di costruttori e maestranze greche, che operavano in sinergia con altre compagini etniche all'interno dei grandi cantieri dell'età medio-tardo repubblicana. Tradizioni che poi si sarebbero ben radicate in una società ormai pienamente romanizzata nelle epoche successive.

Purtroppo però, non avendo ancora una conoscenza abbastanza consolidata della distribuzione geografica delle attestazioni archeologiche di tali pratiche nel Mediterraneo - e in particolar modo in Italia - e risentendo di una carenza di testimonianze letterarie precedenti all'età romana che offrano esplicito riferimento all'uso di conchiglie in attività costruttive, non è possibile portare ulteriori indizi a supporto dell'ipotesi precedentemente esposta.

Pur restando quindi aperto un ragionamento "verticale" sulla possibile diffusione della specifica tecnica nel tempo all'interno del bacino del Mediterraneo, un discorso "orizzontale" di migrazione di saperi tra diversi campi di applicazione è più facilmente documentato: come ricorda il passo di Virgilio, l'impiego dei molluschi a funzione "drenante" richiama pratiche sperimentate al suo tempo in ambito agricolo-rustico. Si può quindi supporre una trasposizione di tale tecnica dall'ambito rurale all'ambito costruttivo dell'edilizia, o viceversa. Come visto, nel nord-est italico, dove i principali vitigni si trovavano proprio in aree limitrofe alle coste ${ }^{104}$, la pratica, forse già impiegata nelle campagne col-

101 Per l'Olanda v. Reese, 1979-80, p. 90; per Gaza v. Ronen, 1980, p. 170.

102 BRACCESI, 1977.

103 Bonetto - Previato, 2013; Bonetto, 2015.

104 Cfr. nota 55. 
tivate, deve aver trovato particolare fortuna in ambito edilizio in virtù della leggerezza dei gusci di molluschi, caratteristica particolarmente importante laddove si vada a costruire su terreni cedevoli.

Concludendo, appare comunque chiaro come la società aquileiese abbia sviluppato le tecniche di smaltimento di grandi masse di conchiglie attraverso forme di reimpiego "intelligente" di tali materiali poveri. Ciò offre ancora una volta prova della perizia ingegneristica e del bagaglio di conoscenze tecniche - seppur fondate su basi empiriche - dei costruttori antichi.

\section{RINGRAZIAMENTI}

Un sentito ringraziamento ai professori J. Bonetto, A. R. Ghiotto e M. Salvadori, che mi hanno permesso di prendere parte alle campagne di scavo presso le Domus di Tito Macro e delle Bestie ferite, dove ho avuto modo di osservare e analizzare questi materiali malacologici ed il loro uso. Un ringraziamento particolare a D. S. Reese per i suggerimenti e il materiale bibliografico fornitomi. 


\section{BIBLIOGRAFIA}

Aït Kaci, A., Drine, A., Fentress, E., Morton, T., Rabinowitz, A., Wilson, A. "The excavations" in E. Fentress, A. Drine, R. Holod (eds.), An Island through Time. Jerba Studies, vol. I, the Punic and Roman Periods, Portsmouth, 2009 (Journal of Roman Archaeology, supp. 71), pp. 213-240.

Alberti, M.E. "La prima porpora: primato etnico o comune patrimonio mediterraneo?", in A. VAnzetti et al. (eds.), Studi in onore di Renato Peroni, Borgo San Lorenzo (Firenze), 2006, pp. 733-736.

Alberti, M.E. "Murex shells as raw materials: purple industry and its by-products. Interpreting the archaeological record”, Kaskal, 5, 2008, pp. 73-90.

Antico Gallina, M. "Bonifiche geotecniche e idrauliche con anfore: teoria e pratica di un fenomeno", Fasti On Line Documenti \& Ricerche, 226, 2011, pp. 1-22.

Arena, M., Marcello, A. Su un traffico d'ostriche nellantica Altino, Venezia, 1960.

Balista, C., Sainati, C. “Ostrea non pectines ad Altino: le evidenze archeologiche”, in G. Cresci Marrone, M. Tirelli (eds.), Produzioni, merci e commerci in Altino preromana e romana, Roma, 2003, pp. 331-346.

BAr-Yosef Mayer, D.E. "Pelecypod beds revisited: Glycymeris in Bronze Age sites", Journal of Israel Prehistoric Society, 35, 2005, pp. 45-52.

Birney, K. Leon-Levy expedition to Ashkelon. Grid 51 - final report 2010, 2010, pubblicazione online.

Blanc, A.C. "Residui di manifatture di porpora a Leptis Magna e al Monte Circeo", in R. BARTocci (ed.), Il porto romano di Leptis Magna, Roma, 1958 (Bollettino del Centro di studi per la storia dell'architettura, 13, suppl.), pp. 187-210.

Bonetto, J. "Le mura”, in F. Ghedini, M. Bueno, M. Novello (eds.), Moenibus et portu celeberrima. Aquileia: storia di una città, Roma, 2009, pp. 83-92.

Bonetto, J. "Diffusione ed uso del mattone cotto nella Cisalpina romana tra ellenizzazione e romanizzazione", in E. Buкоwiecki, R. Volpe, U. Wulf-Rheidt (eds.), Il laterizio nei cantieri imperiali. Roma e il Mediterraneo, Atti del I workshop "Laterizio" (Roma, 27-28 novembre 2014), Firenze, 2015 (Archeologia dell'architettura, XX), pp. 105-113.

Bonetto, J., Centola, V., Novello, M., Ventura, P. "Scavi, documenti e studi per una storia della ricerca ai Fondi Cossar", in J. Bonetto, V. Centola (eds.), Aquileia, Fondi Cossar, I. Scavi, ricerche e studi del passato, Roma, 2017, pp. 19-65.

Bonetto, J., Previato, C., “Tecniche costruttive e contesto ambientale. Le sottofondazioni a sedimenti nella Cisalpina e nel Mediterraneo", in G. CusСіто (ed.), Le modificazioni del paesaggio nell'Altoadriatico tra pre-protostoria ed altomedioevo, Atti della XLIII Settimana di Studi Aquileiesi (Aquileia, 10-12 maggio 2012), Trieste, 2013 (Antichità Altoadriatiche, XLIII), pp. 231-264.

Bonetto, J., SAlvadori, M., (eds.), L'architettura privata ad Aquileia in età romana, Padova, 2012 (Antenor Quaderni, 24). 
Boschetti, C., Bridi, E., Cataldi, G. “Settore 5”, in M. Salvadori (ed.), Aquileia: le indagini archeologiche dell'Università di Padova. Lo scavo della Casa delle Bestie ferite. Le campagne 2014 e 2015, Padova, 2016, pp. 21-25.

BracCesi, L. Grecità adriatica. Un capitolo della colonizzazione greca in occidente, Bologna, 1977.

Bruneau, P. “Contribution à l'histoire urbaine de Délos", Bulletin de Correspondance Hellénique, XCII, 2, 1968, pp. 633-709.

Bruneau, P. "Documents sur l'industrie délienne de la pourpre”, Bulletin de Correspondance Hellénique, XCIII, 1969, pp. 759-791.

Bruneau, P. Délos. Les Mosä̈ques, XXIX, Paris, 1972.

Bruneau, P. "Deliaca, 2, 16. La fabrication de la pourpre à Délos”, Bulletin de Correspondance Hellénique, CII, 1, 1978, pp. 110-114.

Brusin, G. Gli scavi di Aquileia. Un quadriennio di attività dell'Associazione Nazionale per Aquileia: 1929-1932, Udine, 1934.

Brysbaert, A. "Common craftmanship in the Aegean and East Mediterranean: preliminary technological evidence with emphasis on the painted plaster from Tell El-Dab'a, Egypt", Egypt and the Levant, 12, 2002, pp. 95-107.

Brysbaert, A. "Rotating angles in measuring the Aegean Bronze Age. The technology of bronze age painted plaster from the Aegean and Eastern Mediterranean", in K.P. Foster, R. LAffineur (eds.), METRON. Measuring the Aegean Bronze Age. Proceedings of the 9th International Aegean Conference (18-21 April 2002), Liège-Austin, 2003, pp. 167-178.

Brysbaert, A. "Murex uses in plaster features in the Aegean and Eastern Mediterranean Bronze Age", Mediterranean Archaeology and Archaeometry, 7, 2, 2007, pp. 29-51.

Bueno, M., Centola, V. "Le domus di Aquileia e le loro evoluzioni architettonico-funzionali in età tardoantica: $\mathrm{i}$ casi delle domus delle Bestie ferite $\mathrm{e}$ di Tito Macro presso i fondi ex-Cossar", in G. Cuscito (ed.), Costantino il grande a 1700 anni dall" Editto di Milano", Atti della XLIV settimana di studi aquileiesi (Aquileia, 30 maggio - 1 giugno 2013), Trieste (Antichità Altoadriatiche, LXXVIII), 2014, pp. 317-334.

Bueno, M., Centola, V., Ghiotto A.R. "Le domus dei fondi ex-Cossar e delle Bestie Ferite: due esempi di trasformazione delle case aquileiesi in età tardoantica", Aquileia Nostra, LXXXIII-LXXXIV, 2014, pp. 171-181.

Bueno, M., Novello, M., Salvadori, M. "Aquileia (UD). Nuovi pavimenti dalla domus delle Bestie Ferite", in C. Angelelli, C. Salvetti (eds.), Atti del XV colloquio dell'Associazione Italiana per lo Studio e la Conservazione del Mosaico, XV, 2010, pp. 1-15.

Buora, M. "Le mura bizantine di Aquileia", Diadora 26/27, 2013, p. 561-584.

Buora, M. "Ipotesi sul porto bizantino di Aquileia", Studia Universitatis Hereditati, vol. 2/1-2, 2014, pp. 11-21.

ÇAKIrlar, C., Becks, R. “"Murex” dye production at Troia. Assessment of archaeomalacological data from old and new excavations", Studia Troica, 18, 2009, pp. 87-104.

Carannante, A. "Archaeomalacology and purple-dye. State of the art and new prospects of research", in J.J. Cantillo, D. Bernal, J. Ramos (eds.), Moluscos y púrpura en contextos arqueológicos atlántico-mediterráneos. Nuevos datos y reflexiones en clave de proceso histórico. Actas de la III Reu- 
nión Científica de Arqueomalacología de la Península Ibérica (Cádiz, 3-4 dicembre 2012), Cádiz, 2014, pp. 273-282.

Cazzarella, A., Minniti, C., Moscoloni, M., Recchia, G. "Linsediamento dell'età del Bronzo di Coppa Nevigata (Foggia) e la più antica attestazione della produzione della porpora in Italia", Preistoria alpina 40/2004, suppl. 1, Trento, 2005, pp. 177-182.

Ceci, L. "Shell midden deposits as coastal resources", World Archaeology, 16, 1984, pp. 62-74.

Centola, V. "Area II, saggio 4", in J. Bonetto, A.R. Ghiotto (eds.), Aquileia - Fondi ex Cossar. Missione archeologica 2013, Padova, 2013, pp. 28-35.

Claasssen, C. Shells, Cambridge, 1998.

Coutelas, A. Pétroarcheologie du mortier de chaux gallo-romain. Essai de reconstitution et d'interprétation des chaînes opératoires: du matériau au métier antique, Tesi di dottorato, Université Panthéon-Sorbonne - Paris I, 2003.

Di Filippo Balestrazzi, E. "Vino e cultura del vino nell'area orientale", in E. Di Filippo Balestrazzi (ed.), Dalla vite al vino. Antropologia e Storia di una Cultura Enologica nell'Angolo più Orientale della Pianura Veneta, Portogruaro (Venezia), 2004, pp. 1-34.

Di Filippo BAlestrazzi, E. "La porpora e l'Adriatico", Quaderni di archeologia del Veneto, 21, 2005, pp. 194-207.

Dilaria, S. Preparazioni pavimentali e attività di cantiere in due domus di Aquileia. Interpretazione delle fonti, studio dei contesti di scavo, analisi archeometriche, Tesi di laurea magistrale, relatore: prof. J. Bonetto, Padova, 2014.

Dilaria, S. "Dietary consumption and purple-dye production in Roman Aquileia: reusing shell-waste as building material", Archaeo + Malacology group newsletter, 27, 2017, pp. 4-6.

Doumas, C. Thera. Pompei of the Ancient Aegean. Excavations at Akrotiri 196779, 1983, London.

DrINE, A. “Témoignages archéologiques sur les activites halieutiqués de Mennix", in J. NAPOLI (ed.), Ressources et activités maritimes des peuples de l'Antiquité. Actes de colloque international (Boulogne-Sur-Mer, 12-14 maggio 2005), Boulogne-Sur-Mer, 2008, pp. 127-137.

Dunbabin, K.M.D. Mosaics in Greek and Roman World, New York, 1999.

Eerkens, J.W., Lipo, C.P. "Cultural Transmission Theory and the Archaeological Record: Providing Context to Understanding Variation and Temporal Changes in Material Culture", Journal of Archaeological Research, 15, 2007, pp. 239-274.

Fantar, M. Kerkouane: cité punique du Cap Bon (Tunisie), 1, Tunisi, 1984.

Fantar, M. Kerkouane: cité punique du Cap Bon (Tunisie), 3. Sanctuarise et cultes. Société - économie, Tunisi, 1986.

Fentress, E., Drine, A., Morton, T., Ghalia, T. "The town and the port", in E. Fentress, A. Drine, R. Holod (eds.), An Island through Time. Jerba Studies, vol. I, the Punic and Roman Periods, Portsmouth, 2009 (Journal of Roman Archaeology, supp. 71), pp. 131-176. 
Ginouvès, R., Martin, R. 1985, Dictionnaire méthodique de l'architecture Grecque et Romaine, Tome I, Matériaux, techniques de construction, techniques et formes du décor, Rome.

Girod, A. Appunti di archeomalacologia, Sesto Fiorentino, 2015.

Greco, A.V. Virtutes Materiae, il contributo delle fonti latine nello studio di malte, intonaci e rivestimenti nel mondo romano, Sandhi, Ortacesus, 2011.

Hallager, E. "A Waste Deposit From a LBA-shrine in Khania (?)", Aegaeum: Annales d’archéologie égéenne De L'Université De Liège, 22, 2001, pp. 175180.

KARALI, L. "Sea shells, land snails and other marine remains from Akrotiri" in D.A. Hardy, J. Keller, V.P. Galanopoulos, N.C. Fleming, T.H. Druitt (eds.), Thera and the Aegean World III. Vol. II, London, 1990, pp. 410-415.

Karali, L. Shells in Aegean Prehistory, Oxford, 1999.

Karali Giannakopoulos, L. "Testimonianze della produzione della porpora nell'Egeo nell'antichità', Preistoria alpina, 40/2004, suppl. 1, Trento, 2005, pp. 161-166.

Karmon, N., Spanier, E. "Archaeological Evidence of the Purple Dye Industry from Israel", in E. Spanier (ed.), The Royal Purple and the Biblical Blue, Argaman and Tekhelet. The Study of Chief Rabbi Dr. Isaac Herzog on the Dye Industries in Ancient Israel and Recent Scientific Contributions, Jerusalem, 1987, pp. 147-158.

KARDARA, C. "Dyeing and weaving works at Isthmia", American Journal of Archaeology, 65, 3, 1961, pp. 261-266.

Kawerau, G., Wiegand, T. Die Paläste der Hochburg, Berlin, 1930 (Altertümer von Pergamon, 5/1).

Knappett, C., Cunningham, T. "Palaikastro Block M. The Proto - and Neopalatial Town", London, 2012 (British school of archaeology at Athens. Supplementary volumes, 47$)$.

LAW, M. "Mollusc shells from archaeological building materials", in K. SzAbo, C. Dupont, V. Dimitrijevic, L.G. Gastélum, N. Serrand (eds.), Archaeomalacology: shells in the archaeological record, Oxford, 2014, pp. 253-256.

Mac Gillivray, J.A., Sackett, L.H., Driessen, J.M., Hemingway, S. "Excavations at Palaikastro, 1991”, Annual of the British School at Athens, 87, 1992, Atene, pp. 121-152.

Macheboeuf, C., Bolšec Ferri, N., Hanry, A., Katunarić, T. "La pourpre en Istrie”, Mélanges de l'École Française de Rome, 125, 1, 2013, pp. 259-271.

Marzano, A. Harvesting the Sea. The Exploitation of Marine Resources in the Roman Mediterranean, Oxford, 2013.

Maselli Scotti, F., Paronuzzi, P., Pugliese, N. "Sondaggi geognostici per la prospezione geoarcheologica del territorio di Aquileia: il progetto SARA", in C. Zaccaria (ed.), Archeologia senza scavo. Nuovi metodi di indagine per la conoscenza del territorio antico, Atti della XXVIII Settimana di studi aquileiesi (Aquileia, 25 - 28 aprile 1997), Trieste, 1999 (Antichità Altoadriatiche, XLV), pp. 79-97.

Maselli Scotti, F., Rubinich, M. "I monumenti pubblici”, in F. Ghedini, M. Bueno, M. Novello (eds.), Moenibus et portu celeberrima. Aquileia: storia di una città, Roma, 2009, pp. 93-110. 
Merrony, M. W. Socio-economic aspects of Late Roman mosaic pavements in Phoenicia and Northern Palestine, Oxford, 2013 (BAR International series, 2530).

Mynors, R.A.B. Georgica, edizione critica, 1990.

Mozzi, P., Fontana, A., Ferrarese, F., Ninfo, A. "Geomorfologia e trasformazione del territorio", in M. Tirelli (ed.), Altino antica. Dai veneti a Venezia, Venezia, 2011, pp. 13-18.

Pecchioni, E., Fratini F., CANTisAni, E. Le malte antiche e moderne tra tradizione e innovazione, Bologna, 2008.

PedrazzI, T. "La lavorazione della porpora e dei tessuti”, in L.I. Manfredi, A. Soltani (eds.), I Fenici in Algeria. Le vie del commercio tra il Mediterraneo e l'Africa Nera, Bologna, 2011, pp. 115-122.

Perpignani, P., Fiori, C. Il mosaico "non-spazzato". Studio e restauro dell'asaroton di Aquileia, Ravenna, 2012.

Petrucci, G. "Sfruttamento della fauna nel territorio di Aquileia: trasformazione, consumo e distribuzione dei prodotti. I dati dell'archeozoologia", in G. Cuscito, C. ZacCARIA (eds.), Aquileia dalle origini alla costituzione del ducato longobardo: territorio, economia, società, Atti della XXXVII Settimana di Studi Aquileiesi (Aquileia, 18 - 20 maggio 2006), Trieste, 2007a (Antichità altoadriatiche, 65), pp. 755-782.

Petrucci, G. "Analisi dei resti faunistici", in C. Morselli (ed.), Trieste antica, lo scavo di Crosada. I materiali, Trieste, 2007b, pp. 511-559.

Petrucci, G. "Lo sfruttamento delle risorse marine a Trieste in età romana. I dati dai recenti scavi di Crosada di Cavana”, in R. Auriemma, S. Karinja (eds.), Terre di mare. L'archeologia dei paesaggi costieri e le variazioni climatiche, Atti del Convegno internazionale di studi (Trieste, 8-10 novembre 2007), Trieste, 2008, pp. 470-474.

Previato, C. Aquileia. Materiali, forme e sistemi costruttivi dall'età repubblicana alla tarda età imperiale, Padova, 2015 (Antenor Quaderni, 32).

REDINI, P. "I pavimenti a commesso laterizio della Regio VII: un aggiornamento", in F. Guidobaldi, A. Paribeni (eds.), Atti dell'VIII Colloquio dell'Associazione Italiana per lo Studio e la Conservazione del Mosaico (Firenze, 21-23 febbraio 2001), 2001, Ravenna, pp. 227-242.

REESE, D.S. "Industrial exploitation of murex shells: purple-dye and lime production at Sidi Khrebish, Benghazi (Berenice)", Libyan Studies, 11, 19791980, pp. 79-93.

Reese, D.S. "Palaikastro Shells and Bronze Age Purple-Dye Production in the Mediterranean Basin", The Annual of the British School at Athens, LXXXII, 1987, pp. 201-206.

Reese, D.S. "Iron Age Shell Purple-Dye Production in the Aegean", in J.W. Shaw, M.C. Shaw (eds.), Kommos IV. The Greek sanctuary, Princeton, 2000, pp. 643-645.

ReEse, D.S. "Shells from Sarepta (Lebanon) and East Mediterranean Purple-Dye Production", Mediterranean Archaeology and Archaeometry, 10, 1, 2010, pp. 113-141.

Reese, D.S. "The Invertebrates" in E. Hallager, B.P. Hallager (eds.), The Greek-Swedish Excavations at the Agia Aikaterini Square, Kastelli, Khania, 1970-1987, 2001, 2005, and 2008 V The Late Minoan IIIA:1 and II Set- 
tlements, Stockholm, 2016 (Skrifter utgivna av Svenska Institutet $i$ Athen, XLVII, V, 1), pp. 396-417.

Reitz, E.J., Shackley, M.L. Environmental Archaeology, New York, 2012.

Ronen, A. "The origin of the raised Pelecypod beds along the Mediterranean coast of Israel", Paléorient, 6, 1980, pp. 165-172.

Ruscillo, D. "Reconstructing Murex Royal Purple and Biblical Blue in the Aegean Archaeomalacology", in D.E. BAR-Yosef Mayer (ed.), Molluscs in former environments of human behavior, Proceedings of the $9^{\text {th }}$ Conference of the International Council of Archaeozoology (Durham (agosto 2002), Oxford, 2005, pp. 99-106.

Ruscillo, D. "Faunal remains and murex dye production", in J.W. SHAw, M.C. Shaw (eds.), Kommos V. The Monumental Minoan Buildings at Kommos, Princeton, 2006, pp. 776-844.

Salzmann, D. "Mosaiken und Pavimente in Pergamon. Vorbericht der Kampagnen 1989 und 1990", Archäologischer Anzeiger 1991, 1991, p. 433-456.

Salzmann, D. "Zu den Mosaiken in den Palästen IV und V Von Pergamon", in E. Winter (ed.), Studien zum antiken Kleinasien III, Bonn, 1995 (Asia Minor Studien, 16), pp. 101-112.

Schmidt, O. Brehms Tierleben, 10, Leipzig, 1893.

STARINIERI, V. Study of materials and technology of ancient floor mosaics substrate, Tesi di dottorato, Università Alma Mater di Bologna, supervisor: prof.ssa Ioanna Papayianni, 2009.

Stefanidou, M., Papayianni, I., Pachta, V. "Evaluation of inclusions in mortars of different historical periods from Greek monuments", Archaeometry 54, 4, 2012, pp. 737-751.

Tomasello, F., Fontane e ninfei minori a Leptis Magna, Roma, 2005 (Monografie di Archeologia Libica, XXVII).

Trapani, F., "Afro murice. Le malte di murici e la topografia di Leptis Magna in periodo giustinianeo", in R.M. BonacAsa CARra (ed.), Pagani e Cristiani a Sabratha e Leptis Magna tra III e VI secolo d.C. Monumenti e reperti, tradizione e immagini, Atti del seminario di studio (Agrigento, 26-27 gennaio 2012), Palermo, 2012, pp. 215-230.

Westgate, R. "Pavimenta atque emblemata vermiculata: regional styles in Hellenistic mosaic and the first mosaics at Pompeii", American Journal of Archaeology, 104, 2, 2000, pp. 255-275.

Wilson, A.I. "Commerce and industry in Roman Sabratha”, Libyan Studies, 30, 1999, pp. 147-168.

Wilson, A.I. "Marine resource exploitation in the cities of coastal Tripolitania", in M. Khanoussi, P. Rugged, C. Vismara (eds.), L’Africa Romana. Lo Spazio marittimo del Mediterraneo occidentale: geografia storia ed economica, I, Roma, 2002, pp. 429-436.

Wilson, A.I. "Archaeological evidence for textile production and dyeing in Roman North Africa" in C. Alfaro, J.P. Wild, B. Costa (eds.), Purpureae Vestes. Textiles y tintes del Mediterráneo en época romana (Ibiza, 8-10 noviembre 2002), Valencia, 2004, pp. 155-164.

Wilson, A.I., TÉbar Megías, E. "Purple dye production at Hellenistic Euesperides (Benghazi, Libya)", in J. NAPOLI (ed.), Ressources et activités mari- 
times des peuplés de l'antiquité. Actes de colloque international (BoulongeSur-Mer, 12-14 maggio 2005), Boulogne-sur-Mer, 2008, pp. 231-238.

Wilson, A.I., Bennett, P., Buzaian, A.M., Buttrey, T., Fell, V., Found, B., Göransson, K., Guinness, A., Hardy, J., Harris, K., Helm, R., Kattenberg, A., Morley, G., Swift, K., Wootton, W.T., Zimi, E. "Euesperides 2003: Preliminary report on the Spring 2003 Season", Libyan Studies, 35, 2004, pp. 149-190.

Wootton, W. T. "Making and meaning: the Hellenistic mosaic from Tel Dor", American Journal of Archaeology, 116, 2, 2012, pp. 209-234.

Zaouali, J. "Marine and Land Molluscs", in H.R. Hurst (ed.), Excavations at Carthage, The British Mission II/1. The Circular Harbour, North Side. The Site and Finds other than Pottery, Oxford, 1994 (British Academy Monographs in Archaeology, 4), pp. 320-324. 
